Revue d'histoire de l'Amérique française

REVUE D.HISTOIRE DE L'AMÉRIQUE FRANÇAISE

\title{
Les capitaux français et le Québec, 1855-1900
}

\section{Magella Quinn}

Volume 24, numéro 4, mars 1971

URI : https://id.erudit.org/iderudit/303019ar

DOI : https://doi.org/10.7202/303019ar

Aller au sommaire du numéro

Éditeur(s)

Institut d'histoire de l'Amérique française

ISSN

0035-2357 (imprimé)

1492-1383 (numérique)

Découvrir la revue

Citer cet article

Quinn, M. (1971). Les capitaux français et le Québec, 1855-1900. Revue d'histoire de l'Amérique française, 24(4), 527-566. https://doi.org/10.7202/303019ar d'utilisation que vous pouvez consulter en ligne.

https://apropos.erudit.org/fr/usagers/politique-dutilisation/ 


\title{
LES CAPITAUX FRANÇAIS ET LE QUEBEC, 1855-1900
}

\author{
Magella Quinn \\ Collège militaire royal \\ Kingston, Ont.
}

En 1877, plus d'un siècle après la conquête des colonies françaises nord-américaines par l'Angleterre, Arthur Buies rappelle que cet événement a provoqué la rupture complète entre la colonie et son ancienne mère-patrie:

La France ne fut plus pour nous qu'un souvenir. Les navires marchands qui, une fois par année, nous mettaient en communication régulière avec elle, ne reparurent plus; aucun émigrant ne nous arriva de ses ports, pas plus qu'aucun de ses produits; nous connûmes à peine son histoire, et l'écho de ses grandes révolutions nous parvint comme le retentissement de catastrophes inexplicables. Intellectuellement, politiquement, historiquement, le Canada fut séparé de la France... ${ }^{1}$

C'est un fait que la guerre de Sept Ans a abouti à couper toutes relations politiques, économiques et commerciales officielles entre la France et le Canada pendant 100 ans. Mais la rupture ne fut pas aussi totale que le laisse entendre cet auteur.

En effet une partie de l'élite canadienne-française, au cours de la période mentionnée, subira l'influence de la Révolution française, du $19^{\circ}$ siècle libéral et surtout celle des penseurs de ce grand siècle: Veuillot, Lamennais, Lacordaire, Montalembert et quelques autres. Monsieur Claude Galarneau, dans un article paru en $1961^{2}$, nous fait connaître l'existence de relations et de contacts culturels assez importants entre les deux communautés nationales. Des prêtres français, "émigrés de la Révolution", d'anciens soldats de l'Empire, des religieux de différentes

1 Arthur Buies, Question Franco-Canadienne. Construction de navires français au Canada. - Cmmerce de vins avec la France (Montréal, 1877), 3.

2 Claude Galarneau, "Les échanges culturels franco-canadiens depuis 1763", Recherches et Débats du Centre catholique des intellectuels français, cahier no 34 (mars 1961): 68-78. Titre spécial du numéro: Le Canada français aujourd'hui et demain.

[527]

RHAF, vol. 24, no 4 (mars 1971) 
communautés françaises trouvent refuge en terre québécoise et répandent leurs idées. Régulièrement, des étudiants vont acquérir à Paris leur formation professionnelle ou artistique. Enfin, la reproduction abondante d'articles de journaux français, par Le Canadien et La Minerve, fait aussi connaitre les multiples aspects de l'évolution politique, sociale et idéologique de l'élite française. "Quand La Capricieuse remonte le fleuve en 1855, ce sont les relations officielles qui reprennent (...) les rapports culturels franco-canadiens n'ont pas manqué depuis deux siècles et (...) ont été marqués par plus de diversité qu'on ne le répète d'ordinaire ${ }^{3}$."

La participation commune de la France et de l'Angleterre au règlement de la Question d'Orient, leur signature conjointe du traité de Paris en 1856, la disparition des derniers vestiges du mercantilisme et l'application de plus en plus généralisée des théories du libéralisme économique dans le domaine des échanges internationaux, sont autant de facteurs qui peuvent inciter les gouvernements de deux pays à renouer ou à intensifier leurs relations politiques et commerciales. Ce contexte politique et économique, au milieu du $19^{\mathrm{e}}$ siècle, explique en même temps le rapprochement officiel entre la France et le Canada en 1855. On assiste ensuite à la signature de traités commerciaux entre la France, l'Angleterre et les Dominions. C'est dans cet esprit de rapprochement que s'explique l'accueil triomphal que les BasCanadiens réservent à l'équipage de la frégate française $L a$ Capricieuse en $1855^{4}$. Enfin, cette atmosphère favorable incite la France et le Canada à échanger des représentants officiels et fait naître des relations économiques et culturelles entre la France et le Québec, qui s'avéreront intéressantes.

Au cours de cette même période, certains besoins du Québec et quelques secteurs de son économie éveillent l'intérêt d'un petit nombre de détenteurs de capitaux en France et créent un mouvement d'investissements vers le Québec dans la colonisation, le crédit foncier, les prêts d'Etat, l'exploitation de la betterave à sucre et les mines de phosphate. Ces capitaux n'entraînent pas un élan économique de toute première importance pour le Québec, mais leur seule présence nous semble justifier l'analyse que nous vous proposons.

\section{Ibid., 73 et 77.}

4 Voir à ce sujet: Ronald Desmonds Lewis Kinsman, The visit to Canada of "La Capricieuse" and M. le commandant de Belvèze in the summer of 1855 as through the French language press of Lower Canada (Montréal, Thèse de maîtrise, Université McGill, 1959), $176 \mathrm{p}$. 


\section{1 - CONTEXTE POLITIQUE ET ECONOMIQUE}

L'application d'un protectionnisme sévère, une guerre de tarifs sans merci au niveau du commerce international alimentent, au $18^{\circ}$ siècle et pendant une partie du $19^{e}$ siècle, les vieilles rancunes politiques entre différents pays européens. La France et l'Angleterre n'échappent pas à ce mouvement. Cependant vers 1850 , il se manifeste une évolution rapide. Les suites de la révolution industrielle et la mise en pratique, sous l'influence de l'école de Manchester, des théories du libéralisme économique, amènent l'avènement d'une économie de type capitaliste qui se développe ensuite jusqu'à nos jours. La découverte de nouvelles mines de métaux précieux provoque l'abondance de la monnaie sur le marché international: le stock d'or monnayé dans le monde passe, entre 1875 et 1908 , de 7 à 33 milliards de francs. Nous assistons au développement du crédit par la création d'un nouveau système bancaire constitué par les banques d'affaires et les banques de dépôt. De nouvelles habitudes se créent, les emprunts d'Etat deviennent habituels, les sociétés par actions connaissent un essor considérable et les sociétés anonymes se multiplient. La production de masse est rendue possible par l'utilisation de la machine et le progrès du financement. Enfin, dans le commerce, les modes de transport et la distribution de cette énorme production se transforment à l'échelle mondiale.

En 1846, l'Angleterre ouvre la voie du libre-échange dans le domaine du commerce international et la France suit son exemple en 1860. En quelques années, l'ère de l'exclusivité réservant aux métropoles le commerce avec leurs colonies est terminée. Dans ce contexte économique du libre-échange international, et celui de la guerre de Crimée, un événement précis allait marquer la reprise officielle des relations économiques entre la France et le Canada, particulièrement entre la France et le Canada français: l'arrivée, en 1855, de la corvette française La Capricieuse, dans les eaux du Saint-Laurent.

Après la guerre de Crimée, l'Angleterre et la France veulent profiter de la conjoncture favorable pour reprendre officiellement des relations commerciales si longuement interrompues. L'abolition récente par l'Angleterre des anciens tarifs douaniers ${ }^{5}$ qui avaient rendu impraticables les échanges entre le Canada et l'étranger, donne à la France l'occasion de renouer les relations

5 Les droits de douane pour des articles comme les tissus, les bijoux, les parfums ne dépassent pas $12 \%$. Sur les eaux-de-vie et spiritueux cependant, on exige des taxes pouvant atteindre $25 \%$ ad valorem. 
économiques avec son ancienne colonie. Elle peut donc envoyer son porte-parole officiel, Paul-Henri de Belvèze, qui occupe le poste de "commandant des forces françaises dans les eaux de Terre-Neuve" depuis 1853. Il commandera La Capricieuse dans les eaux du Saint-Laurent en $1855^{6}$. Les instructions venant de France à de Belzève précisent que cette mission doit être exclusivement commerciale et sans caractère diplomatique. Les discours chaleureux du commandant annoncent cependant une ère de relations suivies dans tous les domaines entre la France et le Québec, et le but visé est largement dépassé. Le contexte politique et économique de la France, au cours de la $2^{\mathrm{e}}$ moitié du siècle, explique en grande partie les événements précis dont nous allons parler.

\section{En France}

Dans son histoire économique de la France, Henri Sée affirme que la "période de 1852 à 1914 a été marquée par le triomphe du capitalisme, dont l'emprise s'est accentuée de plus en plus dans presque tous les domaines de la vie économique..." L'économie française connaît cependant, au cours de ces années, les variations d'un cycle Kondratieff tout à fait normal. Ces fluctuations correspondent d'ailleurs à la situation internationale d'alors: hausse rapide de 1852 à 1870, stagnation entrecoupée de courtes dépressions brutales entre 1871 et 1895, et finalement nouvelle relance économique de 1896 à 1920.

De 1850 à 1870, le rythme de l'expansion, dans l'économie française, atteint un niveau inégalé jusque-là : la production du charbon et de la fonte ainsi que le commerce extérieur triplent, les importations de coton multiplient par 9 leur volume, et le réseau ferroviaire devient six fois plus long. Et même si cette période connaît quelques fuites de numéraires aux Etats-Unis, en Extrême-Orient, en Crimée, provoquant un ralentissement des investissements intérieurs, et même si à la fin du Second Empire on voit freiner considérablement l'économie en raison de la situation politique européenne de 1870, le progrès, par rapport à 1850, est cependant très important.

Les débuts de la Troisième République sont sous le signe de l'instabilité monétaire due à la guerre de 1870-71 et aux

6 Pour un survol rapide du sujet, voir l'article d'Armand Yon, "Les Canadiens français jugés par les Français de France 1830-1939", RHAF, 18, no 4 (mars 1965) : 517-533.

7 Henri Sée, Histoire économique de la France. Les temps modernes 1789-1914 (Paris, Armand Colin, 1942), 245. 
suites de la Commune. Malgré une petite remontée sous la poigne solide de Thiers avant 1878, la période allant jusqu'à 1895 en sera une de stagnation et même de courtes crises brutales en 1882 et en 1889. Les facteurs favorables à cette époque, du moins jusqu'en 1882, sont la mobilisation des capitaux (la richesse de la France passant de 105 milliards à 209 milliards de francs entre 1853 et 1878), le rôle nouveau des banques classiques qui participent plus que jamais aux affaires privées et étatiques, et la création de nouveaux types de banques dans le crédit foncier et mobilier.

D'autre part, le commerce international connaît la faveur de Napoléon III. Le traité de commerce signé par la France et l'Angleterre en 1860 le prouve assez bien. C'est la fin d'une longue guerre commerciale qui s'appuyait sur les principes du mercantilisme, poursuivie depuis si longtemps entre les deux pays. Et en fait, même si cette entente connaît quelques altérations sous la Troisième République à cause des tendances protectionnistes de Thiers, et également en raison des fluctuations économiques internationales surtout après 1882, les droits de 1860 seront toutefois en grande partie maintenus.

Dans la seconde moitié du $19^{e}$ siècle, surtout sous la Troisième République, l'empire colonial français subit aussi des transformations majeures. Depuis l'abolition de l'esclavage en 1848 , les anciennes colonies ont perdu de l'importance. L'Algérie, le Sénégal, l'Indonésie, la Tunisie attirent maintenant les capitaux français. En 1870, le territoire colonial français draine déjà 510 millions dans le domaine des affaires. De 1881 à 1913, à la suite du traité de Bardo $^{8}$, le commerce avec la Tunisie passe de 27 à 323 millions de francs. Les échanges s'accroissent aussi avec l'Indochine, Madagascar, l'Afrique occidentale et le monde en général.

Enfin, au cours de cette même période, la France devient un important pays créancier. Son portefeuille à l'étranger passe de 2 à 10 milliards entre 1850 et 1860 , et à 15 milliards en 1880 . La crise économique qui commence en 1882, la baisse du taux d'intérêt qu'elle entraîne en France poussent les Français à se tourner vers les valeurs étrangères rapportant de plus gros intérêts. Les placements annuels à l'étranger passent de un demimilliard en 1886 à un milliard en 1897. Chez les Français, les

8 Traité de Bardo, signé le 12 mai 1881. Il cède à la France le droit d'occuper, en Tunisie, certains points, jusqu'à ce que l'administration locale "fut en état de garantir le maintien de l'ordre". 
affaires industrielles étrangères et surtout les emprunts d'Etat trouvent une importante clientèle.

\section{Le Québec}

En premier lieu on peut s'interroger sur la situation économique du Québec vers 1850. Monsieur Fernand Ouellet nous donne cette réponse:

Les sociétés d'agriculture, qui groupaient Canadiens français et Britanniques, étaient tiraillées par les oppositions nationales (...) A l'heure de l'émigration massive des Canadiens français vers les Etats-Unis, la crise de l'agriculture avait une signification encore plus vitale qu'autrefois aux yeux des élites, en particulier aux yeux de celles qui cherchaient à protéger l'héritage moral du passé et à poursuivre la fameuse lutte pour la survivance ${ }^{9}$.

De plus il faut ajouter qu'entre 1840 et $1850,30,000$ Canadiens français s'orientent vers les Etats-Unis. Au cours de la même décennie, le commerce du bois, un des plus importants secteurs de l'économie québécoise, fait face à deux crises graves. La construction navale est affectée par la baisse des prix et par la naissance du syndicalisme. Enfin, l'abolition du tarif préférentiel sur le blé rétablit le jeu de la libre concurrence, donne à l'Ouest la place avantageuse qui lui revient sur le marché international et force ainsi le Québec à se trouver de nouveaux débouchés ${ }^{10}$.

Les changements structurels de l'économie québécoise, vers les années 50 , nous amènent à considérer des facteurs importants pour les décennies suivantes. La mécanisation et la spécialisation dans les campagnes libèrent une partie de la main-d'œuvre agricole. Le manque de bonnes terres prêtes à cultiver et les gros salaires payés par les manufactures de la Nouvelle-Angleterre, attirent de nombreux Canadiens français qui végètent dans les campagnes québécoises. Seule une restructuration de l'agriculture peut alors tirer le Québec de cette situation précaire. Selon les responsables québécois de l'époque, cette transformation est liée à l'immigration d'Ecossais compétents, et son extension

${ }^{9}$ Fernand Ouellet, Histoire économique et sociale du Québec (17601850) (Montréal et Paris, Fides, 1966), 462.

10 Avant l'abolition du tarif préférentiel anglais, le Québec, autant que l'Ouest, pouvait vendre ses produits agricoles à l'Angleterre. Avec l'abolition, l'Ouest, qui produit son blé à des conditions plus avantageuses que le Québec, élimine ce dernier sur le marché international. 
à celle de colons français et belges prêts à défricher de nouvelles terres en fondant des paroisses. La force du Québec au sein de la Confédération y gagnerait à cette politique. Car tout compte fait " 300 colons égalent une paroisse, 30 paroisses égalent un comté, cinq comtés égalent un diocèse, soit un évêque à Rome et cinq députés à Ottawa ${ }^{11 " . ~}$

En deuxième lieu, nous nous demanderons quelles sont les circonstances qui permettront, de 1850 à 1900 , les échanges, les rapports économiques que nous allons situer. Un tableau synoptique expliquant les principales fluctuations de l'économie québécoise fournira les éléments nécessaires à certaines comparaisons au cours de ce travail.

Il est évidemment difficile de retracer entre 1850 et 1900 une politique commune de développement entre la France et le Québec. En fait, les investissements français au Québec sont marginaux et accidentels; ils ne font partie d'aucun programme général d'exportation de capitaux français à l'étranger.

Cependant, la conjoncture politique de la France et du Québec au cours de cette période, les changements structurels et les situations qu'ils provoquent incitent les responsables, au sein des deux gouvernements, à renouer et à intensifier leurs relations économiques et commerciales. La France augmente son commerce international, exporte ses capitaux et nomme des représentants un peu partout dans le monde. De son côté le Canada doit adapter son économie aux lois difficiles du libreéchange international. A l'intérieur de ce Canada, les Québécois cherchent une solution à l'hémorragie démographique qui les afflige; ils cherchent de nouveaux débouchés pour leurs produits agricoles qui subissent la concurrence de l'ouest du pays et celle de l'extérieur; ils tentent aussi d'ouvrir d'autres marchés à leur production forestière et essaient de trouver des capitaux pour réorienter leur économie. Pour obéir aux exigences de ce contexte, la France envoie ses consuls au Canada à partir de $1859^{12}$; le Canada et le Québec enverront leurs représentants officiels en France quelques années plus tard.

\section{2 - LA COLONISATION}

La politique régissant la colonisation québécoise s'élabore à partir des années 1840. En 1842, à la suite de pressions faites

11 Jean Hamelin, "Québec et le monde extérieur 1867-1967", Annuaire statistique du Québec 1968-1969, 6.

12 De 1859 à 1894, le consul réside à Québec. En 1894, il déménage à Montréal. 
TABLEAU DES PRINCIPALES FLUCTUATIONS ÉCONOMIQUES AU QUÉBEC (1851-1901)

\begin{tabular}{|c|c|c|}
\hline & $\begin{array}{l}\text { urbe } \\
\text { lique }\end{array}$ & Description \\
\hline \multirow[b]{2}{*}{$\begin{array}{c}1847 \\
\grave{a} \\
1857\end{array}$} & $\begin{array}{l}\text { Contraction: } \\
1847 \text { à } 1851\end{array}$ & $\begin{array}{l}\text { - Disparition des tarifs protectionnistes anglais. } \\
\text { - Peur de l'annexion aux Etats-Unis. Incerti- } \\
\text { tude, insécurité face à l'avenir. }\end{array}$ \\
\hline & $\begin{array}{l}\text { Expansion: } \\
1851 \text { à } 1857\end{array}$ & $\begin{array}{l}\text { - La plus grande période de prospérité depuis } \\
\text { le début du siècle: exportation croissante de } \\
\text { bois et céréales aux Etats-Unis, construction } \\
\text { ferroviaire, amélioration des canaux, naissance } \\
\text { de banques... } \\
\text { - La seule ombre au tableau: mauvaise récolte } \\
\text { de } 1854 \text { et baisse de l'exportation du bois en } \\
\text { Angleterre en 1855, à cause de la guerre de } \\
\text { Crimée. }\end{array}$ \\
\hline \multirow{2}{*}{$\begin{array}{c}1858 \\
\grave{a} \\
1867\end{array}$} & $\begin{array}{l}\text { Contraction: } \\
1857 \text { à } 1862\end{array}$ & $\begin{array}{l}\text { 1857, l'affaire de l'Ohio Life Insurance Co. et } \\
\text { la crise financière qu'elle cause aux Etats-Unis } \\
\text { et en Angleterre poussent les banques québé- } \\
\text { coises à une prudence qui restreint le crédit et } \\
\text { fait monter le taux d'intérêt. Principaux sec- } \\
\text { teurs de notre économie affectés. } 1857 \text { est } \\
\text { mauvaise récolte au Québec. }\end{array}$ \\
\hline & $\begin{array}{l}\text { Expansion: } \\
1857 \text { à } 1862\end{array}$ & $\begin{array}{l}\text { - La guerre civile américaine favorise nos ex- } \\
\text { portations et nous incite à développer certains } \\
\text { secteurs de notre économie pour nous suffire: } \\
\text { les textiles par exemple. }\end{array}$ \\
\hline \multirow{2}{*}{$\begin{array}{c}1868 \\
\grave{a} \\
1873\end{array}$} & $\begin{array}{l}\text { Contraction: } \\
1868 \text { et } 1869\end{array}$ & $\begin{array}{l}\text { - Fin du commerce du bois équarri avec l'Angle- } \\
\text { terre. Faut se réorienter: diminuer bois } \\
\text { équarri et augmenter la production du bois de } \\
\text { sciage pour le marché américain. La fin de la } \\
\text { guerre civile américaine et difficultés an- } \\
\text { glaises font baisser de } 60 \% \text { les prix de la } \\
\text { farine et du blé. }\end{array}$ \\
\hline & $\begin{array}{l}\text { Expansion: } \\
1870 \text { à } 1873\end{array}$ & $\begin{array}{l}\text { - Les économies américaine et anglaise sont fa- } \\
\text { vorables. Le Québec essaie de se spécialiser } \\
\text { dans la betterave à sucre, l'industrie laitière, } \\
\text { l'élevage, la culture du foin. Au port de Mont- } \\
\text { réal, les importations augmentent de } 45 \% \text { et } \\
\text { les exportations de } 63 \% \text {. La construction ma- } \\
\text { nufacturière augmente. Les prix des produits } \\
\text { agricoles augmentent de } 20 \text { à } 30 \% \text {. }\end{array}$ \\
\hline
\end{tabular}




\begin{tabular}{|c|c|c|}
\hline \multicolumn{2}{|c|}{$\begin{array}{l}\text { Courbe } \\
\text { cyclique }\end{array}$} & Description \\
\hline \multirow{2}{*}{$\begin{array}{c}1874 \\
\text { à } \\
1882\end{array}$} & $\begin{array}{l}\text { Contraction: } \\
1874 \text { à } 1879\end{array}$ & $\begin{array}{l}\text { - } 1873 \text { : crise internationale bancaire. Répercus- } \\
\text { sion dans le monde à cause de surproduction. } \\
\text { - Au Québec, répercussions: commerce baisse, } \\
\text { restrictions bancaires, faillites dans industries } \\
\text { à cause du dumping américain. }\end{array}$ \\
\hline & $\begin{array}{l}\text { Expansion: } \\
1880 \text { à } 1882\end{array}$ & $\begin{array}{l}\text { - Expansion mitigée. Augmentation de nos ex- } \\
\text { portations de produits laitiers et de bétail et } \\
\text { de notre bois à l'Angleterre. }\end{array}$ \\
\hline \multirow{2}{*}{$\begin{array}{l}1882 \\
\grave{a} \\
1887\end{array}$} & $\begin{array}{l}\text { Contraction: } \\
1882 \text { à } 1885\end{array}$ & $\begin{array}{l}\text { Janvier 1882: faillite de Union Générale en } \\
\text { France. Crise bancaire internationale. L'An- } \\
\text { gleterre et les Etats-Unis sont touchés et nos } \\
\text { exportations de bétail, de bois, de produits } \\
\text { agricoles baissent. Les cultivateurs n'ont plus } \\
\text { d'argent et crise dans notre industrie textile. }\end{array}$ \\
\hline & $\begin{array}{l}\text { Expansion: } \\
1885 \text { à } 1887\end{array}$ & $\begin{array}{l}\text { - Reprise économique: tarifs protectionnistes de } \\
1879 \text { font effet, nos produits laitiers deviennent } \\
\text { nos produits d'exportation no } 1 \text { et notre bois } \\
\text { de sciage est très en demande dans "Midde } \\
\text { West " américain. }\end{array}$ \\
\hline \multirow{2}{*}{$\begin{array}{c}1888 \\
\grave{a} \\
1893\end{array}$} & $\begin{array}{l}\text { Contraction: } \\
1888 \text { à } 1890\end{array}$ & $\begin{array}{l}\text { - 1888-1889-1890, récoltes mauvaises ou médio- } \\
\text { cres. Le commerce intérieur est gravement } \\
\text { affecté. } \\
\text { - } 1880-1890 \text { : le monde ouvrier et le monde des } \\
\text { patrons s'organisent; syndicalisme, monopoles, } \\
\text { coalitions... }\end{array}$ \\
\hline & $\begin{array}{l}\text { Expansion: } \\
1890 \text { à } 1893\end{array}$ & $\begin{array}{l}\text { 1891-1892: récoltes abondantes, stimulation de } \\
\text { l'économie dans tous les domaines. Exporta- } \\
\text { tion du beurre et du fromage devient de plus } \\
\text { en plus importante. }\end{array}$ \\
\hline \multirow{2}{*}{$\begin{array}{c}1893 \\
\grave{a} \\
1901\end{array}$} & $\begin{array}{l}\text { Contraction: } \\
1893 \text { à } 1896\end{array}$ & $\begin{array}{l}\text { - Période de spécialisation plus définitive: ex- } \\
\text { portation de produits laitiers et de bois de } \\
\text { pulpe. }\end{array}$ \\
\hline & $\begin{array}{l}\text { Expansion: } \\
1897 \text { à } 1901\end{array}$ & $\begin{array}{l}\text { - Relance de l'économie internationale. Au Qué- } \\
\text { bec, productions forestière et laitière aug- } \\
\text { mentent. }\end{array}$ \\
\hline
\end{tabular}


par Sydenham et Bagot, le gouvernement de Londres accorde à la province de Québec le droit d'élaborer sa propre législation. Cette dernière se sert alors de ses droits et en arrive à une politique de distribution des terres à tout habitant mâle de 18 ans et plus. Entre 1851 et 1901, l'occupation du sol québécois passe de 8 à 14 millions d'acres, soit une augmentation d'environ $78 \%$. Nous devons attribuer à cette nouvelle politique une bonne partie de cette évolution.

Cependant, dès l'application de ce mode de colonisation individuelle, un malaise se fait sentir. Le Courrier $d u$ Canada, le 20 mars 1869 , présente clairement la situation:

Le colon pauvre et habitué à vivre à la journée, ne réussira jamais, en général, à coloniser, à moins qu'il ne soit entouré de personnes ayant des ressources pécuniaires, et qu'il ne s'éloigne pas trop des centres de population. L'expérience prouve que les colons de cette catégorie sont incapables de parvenir à s'établir sur des terres nouveiles, et épuisent inutilement leurs faibles ressources et leurs forces en essayant de défricher des terres qu'ils sont obligés, trop souvent, d'abandonner ou de vendre à vil prix ${ }^{13}$.

Malgré la formation de sociétés de colonisation à partir de 1845 par la classe dirigeante et malgré l'encouragement du gouvernement provincial qui affecte aux intérêts de la colonisation une section des sociétés d'agriculture qu'il crée à partir de 1847, on doit de nouveau repenser à tout ce problème. En 1851, dans le rapport d'un comité spécial sur la colonisation, on affirme: "A moins que la classe nombreuse et intéressante d'agriculteurs ne reçoive de l'aide dans cette province, il faut que le Canada tombe dans la stagnation et l'insignifiance (...) il faut donc que la législature, par tous les moyens en son pouvoir, vienne enfin à son secours ${ }^{14}$."

Ce n'est qu'en 1869 que le gouvernement provincial décide d'œuvrer vraiment. Selon la loi dite des "Sociétés de colonisation", la première société de ce genre légalement formée dans un comté reçoit un subside annuel égal aux contributions prélevées parmi ses membres, et cela jusqu'à concurrence de $\$ 300$. On attribue aussi une somme réduite à $\$ 150$ seulement à la deuxième et à la troisième société de ce même comté. Le département

13 Cité par J.-E. Garon, Historique de la colonisation dans la Province de Québec de 1825 à 1940 (Québec, 1940), 60.

14 Ibid., 1. 
des terres confie à chacune de celles-ci une partie de canton et $\mathrm{y}$ fait effectuer le défrichement qu'il porte à leurs frais. Après trois ans, le domaine est divisé entre les souscripteurs qui acceptent de s'en occuper, de le cultiver ou de le faire cultiver par une personne de leur choix.

Le fonctionnement, l'acquisition et la distribution des terres, les souscriptions individuelles, sont autant d'éléments pouvant varier à l'intérieur de différentes sociétés de colonisation. Dans le cas des compagnies à capitaux français, il est cependant admis de parler d'un type particulier, de sociétés "indépendantes". Elles constituent de véritables sociétés anonymes dont le but premier est l'exploitation des forêts et l'exportation du bois. La colonisation et le respect des exigences du gouvernement provincial en ce domaine leur permettent d'obtenir, à un prix minime, de grandes superficies de terrain, et ensuite disposer de la main-d'œuvre nécessaire à l'exploitation forestière. La Compagnie de colonisation franco-canadienne, la Société canadienne limitée et la Compagnie de colonisation et de crédit des Cantons de l'Est adoptent cette ligne de conduite.

Comment ces compagnies françaises sont-elles attirées en territoire canadien? Dès 1855, l'Exposition universelle de Paris fournit à notre pays l'occasion d'attirer les regards de la France et autres nations européennes. Le gouvernement canadien, par l'entremise de Jean-Charles Taché, commissaire général du Canada, distribue deux brochures, l'une écrite par Taché ${ }^{15}$ luimême et l'autre par Sterry Hunt ${ }^{16}$. En 1856, dans le rapport qu'il présente au gouvernement ${ }^{17}$, Jean-Charles Taché se dit heureux de l'impression que le Canada a laissée. Un témoignage français tiré de l'histoire de l'Exposition de Paris, confirme le bien-fondé de ce sentiment de fierté: "M. J.-C. Taché a d'ailleurs déployé dans l'accomplissement de son mandat un zèle et une activité vraiment méritoires; on peut dire qu'il a popularisé le Canada en France (...) Son esquisse est une de ces œuvres qui, dans un pays comme le nôtre, atteignent leur but ${ }^{18}$."

15 J.-C. Taché, Esquisse du Canada considéré sous le point de vue économiste (Paris, H. Bossange et Fils, 1855), 180 p.

16 Sterry Hunt, Esquisse géologique du Canada, Chimiste et minéralogiste de la commission géologique du Canada. (Aucun détail).

17 J.-C. Taché, Le Canada et l'exposition universelle de 1855. Rapport de J.-C. Taché, Commissaire du Canada à Paris en 1855 (Toronto, John Lovell, 1856), $477 \mathrm{p}$.

18 Charles Robin, Extrait relatif au Canada, de l'histoire de l'Exposition universelle de 1855 (Québec, E.-R. Fréchette, 1856), 3. 
Jusqu'aux années 1870, la brochure de J.-C. Taché en est le témoignage avec deux autres publications du gouvernement canadien parues en $1860^{19}$ et $1864^{20}$. En 1868, les délégués des provinces et du fédéral s'entendent sur le partage des pouvoirs et des responsabilités dans le domaine de l'émigration ${ }^{21}$. En 1871, le Québec nomme deux agents temporaires pour aller en Europe dans le but "d'attirer vers cette province une immigration agricole". Il s'agit de Edouard-A. Barnard pour le Continent et de M. Jones pour les Iles britanniques. Comme moyen de propagande, M. Barnard distribue une brochure québécoise publiée en 1870, La province de Québec et l'émigration européenne ${ }^{22}$. La conjoncture politique européenne ${ }^{23}$ favorise ses efforts, et au cours des trois années suivantes, on enregistre les plus forts contingents d'immigration française et belge au Canada de 1850 à $1900: 1,366$ en $1872 ; 2,634$ en 1873 et 1,632 en 1874. On constate donc une certaine continuité dans les relations franco-canadiennes depuis les années 1850 et surtout un intérêt croissant des Français pour le Québec, à partir de 1870.

\section{La Compagnie de colonisation franco-canadienne}

Le 26 février 1870, le consul français à Québec, Fred Gauthier, note que la loi de 1869 sur les sociétés de colonisation et que la volonté effective du gouvernement provincial de soutenir ce domaine, ont décidé quelques Français à former une société de colonisation et à prendre en main l'établissement d'une petite colonie de Bretons au Québec, dans le canton Langevin, comté de Dorchester. Le consul espère qu'en mai 1870 on pourrait recevoir ces immigrants ${ }^{24}$. En fait, la première famille française viendra s'installer dans la colonie au début de novembre 1873.

La Compagnie de colonisation franco-canadienne, fondée à Paris au début de 1872, "est organisée en société anonyme, au capital de deux millions de francs, et est administrée par un

19 Gouvernement du Canada, Le Canada, courte esquisse de sa position géographique, ses productions, son climat, ses ressources, ses institutions... (Québec, John Lovell, 1860), 48 p.

20 Stanislas Drapeau, Coup d'cil sur les ressources productives et la richesse du Canada (Québec, Typographie de Léger Bourassa, 1864), $36 \mathrm{p}$.

21 Voir détails dans article de Jean Hamelin, Annuaire du Québec 1968-1969, 7-8.

22 (Gouvernement du Québec, Québec, Imprimerie de L'Evénement, 1870), $142 \mathrm{p}$.

23 La guerre franco-allemande provoque l'émigration française.

24 Archives des Affaires étrangères (à l'avenir AAE), Paris, $C C$, vol. 6 , fol. 069 . 
conseil composé de notabilités honorées et jouissant d'une haute considération dans les affaires en France ${ }^{25}$ ". Il s'agit de M. Eugène Mahieu de Cherbourg, président du Syndicat de bois du Nord en France, M. Fournier, ingénieur civil, M. Peyrusse, secrétaire général des chemins de fer de Charentes, et de M. E. Uzac, de la maison Guz et Compagnie de Bordeaux ${ }^{26}$.

A l'automne 1872, Victor Vannier, petit industriel français, visite pour la première fois la région de la Beauce où il a l'intention de réaliser le projet. Il est en effet le représentant officiel de la Compagnie de colonisation franco-canadienne et l'âme dirigeante de toute l'affaire. Après une visite de 8 jours sur les lieux, il revient enchanté et presse le gouvernement provincial de hâter les procédures. Dans un rapport du 23 octobre 1873 , le commissaire des Terres, Louis Archambault, recommande au lieutenant-gouverneur de bien vouloir accorder l'octroi de 320,000 acres de terre à la Compagnie franco-canadienne.

Les détails des conditions faites à cette compagnie nous indiquent le fonctionnement d'une telle société indépendante de colonisation. Les 320,000 acres de terrains sont choisis dans les lots non occupés des cantons Adstock, Forsyth, Gayhurst, Jersey, Metgermette-nord et Metgermette-sud du comté de Beauce, et des cantons Langevin et Watford du comté de Dorchester. Cette concession est faite en "réserve". Selon cette clause, M. Vannier, ou la société qu'il représente, ne doit entrer en possession du terrain que progressivement, avec l'arrivée annuelle des deux cents familles promises. Le processus, échelonné sur une période de 8 ans, accorde à la compagnie environ 40,000 acres de terre annuellement. Le gouvernement s'engage, aussi longtemps que le nombre exigé de familles entrent dans la colonie, à protéger, sauvegarder les terres désignées à l'avance pour l'installation complète, et d'y refuser toute concession ultérieure. Dans le cas contraire, le gouvernement peut disposer de la partie en "réserve" non encore occupée. Enfin, pour éviter la spéculation, la compagnie n'a jamais le droit d'exiger en son nom, l'émission des lettres-patentes. Cette émission est plutôt faite au nom du colon habitant réellement le lot. M. Vannier devra donc établir, entre 1874 et $1882,1,600$ colons et leur famille, bâtir 1,600 maisons

25 AAE, Paris, CC, 20 octobre 1875, fol. 287. Lettre de M. Vannier datée du 16 octobre 1875 .

26 E. Moreau, "Rapport spécial de M. E. Moreau, directeur de colonisation sur une visite d'inspection dans le comté de Beauce" (Documents de la Session [provinciale] (à l'avenir DSP), 1875), no 4: 341. 
d'au moins 20 pieds par 16 , et faire défricher 32,000 acres de terre, soit un dixième de l'étendue de chaque lot.

A l'automne 1873, M. Vannier et le gouvernement provincial délimitent 65,000 acres de terrain dans le canton de Metgermettenord. Sur ce total, le vendeur se réserve 21,000 acres de "terrains intermédiaires", politique qu'il applique dans chaque canton concédé. Ces terrains prennent de la valeur à la suite d'établissements prospères et garantissent au gouvernement une somme appréciable de revenus. La Compagnie franco-canadienne possède donc 44,000 acres de terre, les seuls d'ailleurs qu'elle se verra attribuer au cours de sa brève existence. Le prix en est fixé "à 20 centimes l'acre, à la charge par les acquéreurs d'ouvrir euxmêmes les chemins rigoureusement indispensables, ce qui, de fait, (remet) le prix de vente au taux ordinaire de trente centimes l'acre, lorsque le gouvernement fait l'ouverture des chemins ${ }^{27}$ ".

En janvier 1874, la Compagnie franco-canadienne émet, sur le marché de Paris, des actions pour un montant de $1,000,000$ de francs. Au Québec et à Paris, l'enthousiasme est grand. La Minerve bénit l'espoir maintenant réel de voir "les capitaux français rivaliser avec les capitaux anglais". Un journal parisien que cite $L a$ Minerve, tous calculs faits, prétend qu'au cours de ses 50 années d'existence, la compagnie réaliserait un produit net de 46,800,000 francs. L'analyse des faits, et non des prévisions, nous rend beaucoup moins enclin à un tel optimisme. La compagnie existera en effet jusqu'en 1878 , sans avoir rencontré les exigences du gouvernement provincial.

Les réalisations matérielles de la compagnie, dans la colonie française de Metgermette, retiennent d'abord notre attention. Trois personnes visitent la colonie en janvier, au cours de l'été et à l'automne de l'année 1874. Il s'agit de MM. Chicoyne et Moreau, agents gouvernementaux, et de Edouard Montpetit. Ce qu'ils rapportent dans leurs écrits ${ }^{28}$ nous permet de suivre le progrès de l'établissement et son état après la dernière de ces trois visites, en automne 1874 .

En quelques mois, voici les projets qu'on a réalisés: un pont à l'embouchure de la rivière Abénaki, un moulin à scie, 14 "constructions" devant servir aux chantiers (menuiserie, hangar à grain, stalles à chevaux, forge...), une maison de

27 Ibid.

28 J.-A. Chicoyne, "Rapport sur l'établissement de la Compagnie Franco-Canadienne dans Metgermette", DSP, 1874-75, no 4, app. no 3. E. Moreau, loc. cit. 
refuge, 13 maisons d'habitation, une glacière, 625 acres de terre défrichée et environ 10 milles de chemin reliant la colonie au monde environnant. Ceci montre bien la détermination des responsables de l'entreprise. La colonie amorce donc un départ relativement prometteur en 1874 et les travaux qui y sont entrepris fournissent un emploi à bon nombre de travailleurs: "De quinze qu'ils étaient d'abord, leur nombre fut bientôt porté à 40, et dans le cours de janvier et février, le chantier comptait 140 hommes de toutes mains, charpentiers, menuisiers, mineurs, forgerons...29."

Les années subséquentes ne portent pas autant de fruits. A la fin de 1874, en 1875 et 1877 , le gouvernement du Québec doit accorder des délais à la compagnie dans l'espoir de la voir remplir ses obligations. En 1877 , les nouveaux actionnaires ${ }^{\mathbf{3 0}}$ ont droit aussi à ces délais. Le gouvernement diminue enfin les exigences concernant la colonisation, mais inutilement. En 1881, un document de la session provinciale indique que le gouvernement a repris possession du canton de Metgermette depuis déjà quelque temps ${ }^{31}$.

$\mathrm{Au}$ cours de ces quelques années, une douzaine de Français seulement se rendent dans la colonie. On peut s'interroger sur les motifs qui ont réduit ainsi la venue des colons et se demander les raisons de la faillite de la Compagnie de colonisation francocanadienne. Il semble bien que la rigueur du climat a pu en décourager un certain nombre; le manque de chemins d'accès peut être aussi une autre raison importante. Cependant, dans une lettre du 13 octobre 1875 qu'il adresse à M. Lefaivre, consul de France à Québec, Victor Vannier nous fournit la véritable raison: certains arrêtés français de novembre et décembre 1873 interdisent d'engager des colons en France et de les amener habiter la colonie française de Metgermette ${ }^{32}$. En 1884, Frédéric Gerbié attire aussi l'attention sur le fait que l'opposition du gouvernement français à l'immigration est la cause principale qui a contribué à l'insuccès de chacune des tentatives de colonisation ${ }^{33}$. Enfin, la plupart des consuls français dans notre pays appuient cette politique de leur gouvernement et contribuent

29 Montpetit, Colonie française de Metgermette, 9.

30 En 1877, les actionnaires abandonnent leurs droits et privilèges à M. Mahieu de Cherbourg qui s'adjoint ensuite Adolphe Ouimet, avocat, et J.-B. Rouillard, métallurgiste, tous deux de Montréal.

31 DSP, 1881-82, vol. 15 (1), doc. no 2, app. no 3: 489.

32 AAE, Paris, CC, 20 octobre 1875, fol. 285.

38 Op. cit., 399 et 434. 
ainsi à l'échec de la Compagnie de colonisation franco-canadienne à Metgermette.

Il est intéressant de noter qu'entre 1872 et $1876,6,450$ Français et Belges viennent au Canada avec l'intention de s'y établir. Ces immigrés ne font cependant pas partie de cette précieuse classe rurale dans laquelle $M$. Vannier veut recruter ses colons. Ce sont surtout des ouvriers et des réfugiés politiques qui fuient la France à la suite de la guerre franco-allemande de 1870-71. Le gouvernement français permet à ses ruraux d'émigrer en Algérie seulement où ils peuvent continuer leur travail et contribuer à la prospérité française. Ce gouvernement considère en effet qu'il n'a que faire de colons "qu'on envoie dans un pays dont une partie de la population émigre pour aller chercher aux Etats-Unis les moyens d'existence qui lui manquent chez elle $\mathbf{3 4}^{34}$. Cette immigration ne peut donc fournir, au cours de cette période, la population dont $M$. Vannier a grandement besoin pour rencontrer les exigences du gouvernement du Québec.

\section{La Société Canadienne Limitée}

Dans l'introduction d'une brochure qu'elle publie en $1872^{35}$, la Société générale forestière de France exprime son désir de venir en aide "aux millions de Français" qui veulent quitter la France à la suite de la crise politique et économique que le pays connaît au début de cette décennie. En 1871 déjà, un agent de cette société est venu au Canada pour s'informer des conditions générales de la colonisation, et son rapport favorise le Québec comme région idéale d'immigration. La politique généreuse de concession des terres et la population d'expression française constituent, à son avis, deux facteurs très importants dont doivent tenir compte les futurs émigrants français.

Cette brochure affirme ensuite qu'un arrêté en Conseil du gouvernement québécois lui a accordé, le 15 décembre 1871, une réserve de 320,000 acres de terre dont une partie se trouve dans la Beauce et l'autre dans la vallée de la Matapédia. En fait, les conditions faites à cette compagnie par le gouvernement du Québec sont identiques à celles de la Compagnie franco-cana-

34 AAE, Paris, $C C, 15$ octobre 1873, fol. 439.

35 Société Canadienne Limitée. Pour favoriser la Colonisation au Canada et le développement des relations industrielles et commerciales entre l'Europe et l'Amérique. Notice sur le Canada. Renseignements sur la Province de Québec et les richesses des concessions de la Société Canadienne. Conditions de colonisation (Paris, Imprimerie centrale des chemins de fer, 1872), 2. 
dienne ${ }^{36}$. Malheureusement la Compagnie Canadienne Limitée (c'est sous ce titre que la Société générale forestière de France se fait représenter au Canada) ne réalise aucun de ses projets de colonisation et, avant tout, d'exploitation forestière. Sans parler des raisons de cet échec, M. Pierre Fortin, dans un rapport qu'il soumet au gouvernement du Québec à titre de commissaire des terres de la Couronne, signale que la compagnie n'a pas respecté ses exigences et qu'il s'est vu forcé d'annuler la réserve faite en sa faveur ${ }^{37}$.

\section{La Compagnie de colonisation et de crédit des Cantons de l'Est}

En 1880, les sociétés de colonisation de St-Hyacinthe et de Bagot s'occupent déjà d'attirer les Canadiens rapatriés des Etats-Unis, vers des régions nouvelles, à la conquête de terres neuves. Elles décident cependant d'élargir le champ de leurs activités. Dans ce but, l'agent de colonisation et promoteur des deux sociétés, J.-A. Chicoyne, passe en Europe afin de se rendre compte des possibilités d'attirer des Français et des Belges au Québec. En France, une personne en particulier partage l'enthousiasme de M. Chicoyne pour ce projet. Il s'agit du révérend Père Eugène-Marie Peigné, chanoine honoraire de Nantes. Cet homme possède des biens de famille et désire participer au financement de l'entreprise. Il devient donc bailleur de fonds de la future société et exige en retour la présence au Canada de son neveu, Eugène Bécigneul, pour surveiller les opérations.

Le 30 juin 1881, la législature provinciale du Québec sanctionne l'acte d'incorporation de la Compagnie de colonisation et de crédit des Cantons de l'Est. Afin de réaliser ses buts, c'est-à-dire la colonisation, l'exploitation de nos richesses naturelles et le prêt aux personnes voulant collaborer au développement de la compagnie, cette société se porte acquéreur de trois étendues de terrain. A l'extrémité nord du lac Mégantic, dans la ville actuelle du même nom, elle achète 37 acres où elle a l'intention de construire un moulin à scie et à farine. Au sud du lac, les 106 acres qu'elle possède doivent lui servir à la création d'une station de bateaux à vapeur. Enfin, dans le canton de Woburn, de chaque côté de la rivière Arnold, à environ six milles du lac Mégantic, elle entre en possession de 13,716 acres de terre boisée "pour y établir une nouvelle paroisse francaise qui pourrait devenir le point de départ de plus vastes opérations ${ }^{38 "}$.

36 DSP, 1872, no 2, app. no 20: 23.

37 DSP, 1873, no 2: VIII.

38 Alphonse Cauchon, Lac Mégantic: La Compagnie nantaise; le chemin de fer (1879-1936) (Sherbrooke, 1936), 6. 
Dès l'automne de 1881 , les principaux travaux d'aménagement, c'est-à-dire l'achat même du terrain, la construction de routes et de bâtiments pour les travailleurs, engloutissent les premiers vingt mille dollars investis par l'abbé Peigné dans l'entreprise. Dès novembre de la même année, ce dernier sollicite donc "le clergé et les amis" du diocèse de Nantes afin de porter à $\$ 50,000$ le capital de la compagnie et ainsi parvenir à se lancer dans l'exploitation forestière, but ultime de la compagnie ${ }^{39}$. En 1884, seulement trois ans après le début des opérations, les réalisations peuvent déjà nous surprendre. Le Moulin Nantais, l'usine à vapeur qu'on avait projetée, existe depuis deux ans et peut fabriquer plus de six millions de pieds de bois par année. La première colonie, Channay, est en voie d'organisation dans le canton de Woburn; une ferme appartenant à la compagnie, sur laquelle on cultive 100 acres de terre, une église et un noyau de population y créent une certaine activité.

A chaque année, entre 1885 et 1890 , l'inquiétude des actionnaires français ne cesse de croître. Ils reçoivent en effet des nouvelles de façon irrégulière de la part des responsables canadiens et doivent à quelques reprises confier à différents membres de la famille Bécigneul la surveillance et l'administration de l'affaire. Jusqu'en 1890, ils investissent environ $\$ 60,000$ dans cette région de Mégantic. Cependant, au cours de cette même année, devant le désir des responsables de Channay d'ériger une fromagerie moderne, constatant l'achat de matériel dispendieux que ceux-ci ont importé d'Europe pour réaliser ce projet, les actionnaires prennent peur et cèdent l'affaire pour une somme de $\$ 12,000$ à Frank Dudley, un marchand de bois de Portland.

La compagnie change donc de mains, mais continue ses opérations et ne cesse de jouer un certain rôle économique dans la région. Une douzaine de familles françaises et un bon nombre de familles canadiennes-françaises étaient venues s'établir dans la région pour travailler dans cette entreprise. Il est évidemment difficile de connaître le nombre exact de personnes ou de familles que le Moulin Nantais a pu attirer à Mégantic même. Nous savons cependant qu'en 1871, une cinquantaine de familles y demeurait de façon permanente alors qu'en 1890 on compte 1,173 personnes. L'établissement du Moulin Nantais a vraisemblablement attiré une bonne partie de cette nouvelle population.

${ }^{39}$ Les détails sur le sujet se trouvent dans la brochure d'Alphonse Cauchon ci-haut mentionnée. 29 p. 
Selon J.-E. Garon, cette compagnie, qui vendait à des colons, ou mettait elle-même en culture les terres déboisées par son propre travail "modifia le système de colonisation en ce sens qu'au lieu de laisser les colons isolés, sans direction et sans protection, elle les organisa par groupes afin de fonder des paroisses d'après un plan d'ensemble méthodique et bien arrêté ${ }^{40 "}$.

L'étude de ces trois tentatives françaises dans les domaines de la colonisation et de l'exploitation forestière nous amène aux conclusions suivantes. Nous constatons d'abord une certaine évolution dans les exigences du gouvernement provincial envers ces nouvelles sociétés. Dans le cas de la Compagnie de colonisation franco-canadienne, nous sentons que l'immigration constitue la préoccupation première du gouvernement et qu'il s'attend à une politique nettement orientée vers ce domaine. En 1881, dans la charte de la Compagnie de colonisation et de crédit des Cantons de l'Est, les responsables québécois insistent sur l'aménagement de nouveaux territoires et sur les avantages que ce développement pourrait apporter, non pas aux immigrants, mais aux Québécois eux-mêmes.

Est-ce que l'étude de ces deux seules compagnies de colonisation peut nous permettre de conclure à une telle évolution ? L'échantillonnage est évidemment restreint et les conclusions qu'on en tire peuvent être taxées de simples coïncidences. La conjoncture de l'époque semble cependant appuyer notre hypothèse. En effet, à partir de 1873, l'immigration cesse d'être une priorité pour le Québec. La construction de chemins de fer, qui rend possible l'ouverture de nouvelles régions à la colonisation et en même temps l'absorption du surplus démographique des paroisses rurales, popularisent beaucoup plus les intérêts. D'ailleurs, en 1875, les provinces reconnaissent au pouvoir central l'entière responsabilité de la propagande d'immigration. Ottawa tentera par la suite d'attirer les Européens, Français ou autres, vers l'ouest du Canada. Il est donc logique que le gouvernement québécois ait accordé, vers 1880 , plus d'importance à la redistribution de ses propres effectifs en colons, qu'à la venue d'immigrants français.

Enfin, ces tentatives n'ont pas été vaines. Après 1890, la région du lac Mégantic a continué à se développer en partie grâce à cette industrie du bois créée au cours de la décennie précédente. L'archidiocèse de Québec, après l'échec de Victor

${ }^{40}$ Garon, Histoire de la colonisation..., 85. 
Vannier à Metgermette, envoie des Canadiens français s'y établir afin de consolider les bases déjà posées.

\section{3 - EMPRUNTS PROVINCIAUX - LE CREDIT FONCIER FRANCO-CANADIEN}

Jusqu'ici, les tentatives que nous avons étudiées n'ont pas été couronnées de succès. Deux champs d'action ont cependant permis à quelques investisseurs français des placements avantageux dans notre province : ce sont les emprunts d'Etat et le crédit foncier.

\section{Les emprunts québécois}

En 1879, le continent entier traverse une crise économique, générale aux Etats-Unis, surtout commerciale au Canada. A Ottawa, John A. Macdonald a repris le pouvoir et essaie laborieusement d'appliquer une politique protectionniste envers l'industrie canadienne naissante. A Québec, Joseph-Adolphe Chapleau devient premier ministre et son gouvernement hérite de deux grands problèmes : l'achèvement de la construction des chemins de fer déjà commencés et le renouvellement des obligations de la province.

Pour financer les projets des tronçons ferroviaires QuébecLac St-Jean et Ottawa-Montréal-Québec, le gouvernement avait emprunté $\$ 4,000,000$ à Londres en 1874. Quatre ans plus tard, à cause de la réticence ${ }^{41}$ du marché anglais, le premier ministre Joly s'adresse plutôt à New York et contracte un nouvel emprunt de $\$ 3,000,000$. En 1880, avant de convoquer les députés, Chapleau veut annoncer quelques résultats tangibles et montrer qu'il a déjà trouvé les solutions aux problèmes de l'heure. Pour mettre d'abord la trésorerie à l'aise, il veut contracter un emprunt à long terme de $\$ 4,000,000$. Mais avant d'y réussir, il doit parer aux besoins les plus pressants et trouver du crédit à court terme dans les banques québécoises. En avril 1880, $\$ 550,000$ lui sont accordés par ces institutions. Chapleau peut ainsi se permettre d'attendre les résultats des négociations qu'il a entreprises sur le marché de Paris.

Ce n'est pas sans hésitation que Chapleau choisit de faire appel au marché parisien. En effet, Boucherville avait voulu emprunter la même somme de $\$ 4,000,000$ en 1876 et avait rencontré

41 La dépression économique qui sévit depuis 1873 explique en partie cette attitude. La campagne de dénigrement contre le Québec que les magnats du Grand Tronc ont entreprise à Londres, dans le but d'éviter l'apparition de compagnies rivales, est aussi un facteur important. 
l'opposition du gouvernement français ${ }^{42}$. J.-S.-C. Würtèle, député de Yamaska, représente donc le gouvernement québécois dans cette affaire et se rend à Paris. En avril 1880, il rencontre les grandes personnalités du monde financier et étudie toutes les facettes de l'affaire. Le 8 mai suivant, "après plusieurs pourparlers", il conclut un arrangement avec la Maison L. et $R$. Cahen d'Anvers et Compagnie et fait exécuter les contrats trois jours plus tard. La province de Québec reçoit donc $\$ 4,000,000$ pour lesquels elle s'engage à créer quarante mille obligations de 500 francs chacune au taux d'intérêt de $4.5 \%$ et remboursables au pair le premier juillet 1910. Le terme est donc de 30 ans à compter du premier juillet 1880 , ou de 39 années, comprenant l'intérêt et le fonds d'amortissement. Les paiements doivent se faire à Londres et être ensuite transmis à Paris à la Banque de Paris et des Pays-Bas ${ }^{43}$.

Sur le marché de Paris, le public hésite un court moment avant de souscrire. Cela peut s'expliquer par le fait que les obligations sont lancées sur le marché en France en pleine saison morte pour les opérations financières, dans la deuxième quinzaine de juillet. Le Figaro et Le Gaulois font peut-être aussi vibrer un peu trop la corde sentimentale et patriotique au moment du lancement; l'affaire semble être une œuvre de bienfaisance et par conséquent, elle fait un peu peur aux hommes d'affaires. Mais une fois ce nuage dissipé, l'emprunt est endossé complètement par le public.

En 1890, la situation financière du gouvernement Mercier exige de nouveau le recours à un emprunt. Une dette flottante de plus de dix millions de dollars, le besoin de crédits pour les chemins de fer, les questions des "Biens des Jésuites" et des travaux publics à travers la province ne laissent en effet à la Chambre aucune autre possibilité. A cette occasion, le gouvernement reçoit la permission, grâce au projet de loi 169 , de se procurer une somme "n'excédant pas en total dix millions de piastres et, à émettre à cette fin, des obligations ou débentures portant un intérêt annuel de pas plus de quatre pour cent... 44"

Le fait qu'en 1890, le taux d'intérêt français soit de près de $2 \%$ plus bas qu'en Angleterre ${ }^{45}$, constitue certainement un facteur important dans la décision du premier ministre Mercier

42 AAE, Paris, $C C, 7$ juin 1876, fol. 106.

43 Toute la correspondance au sujet de cet emprunt est contenue dans les DSP de 1880 , no 44.

44 DSP, 1892, doc. no 53: 9-13.

$45 \mathrm{Il}$ est de $3 \%$ en France et de $4.69 \%$ en Angleterre. 
de lancer un nouvel appel au marché de Paris. Une très mauvaise publicité l'oblige aussi à fuir celui de Londres. Ayant l'intention d'effectuer un emprunt provincial à long terme à 3 ou $31 / 2 \%$, il se rend donc à Paris avec son trésorier, Joseph Shehyn. Cependant, dénigré par le comte de Turenne, consul français à Québec, qui l'accuse auprès du gouvernement français d'avoir une politique démesurée de travaux publics ${ }^{46}$, et faisant face à une conjoncture française défavorable puisque “...la grande entreprise de Panama s'achevait en scandale, après avoir drainé l'épargne française pendant 6 ou 7 ans (et qu'un) projet d'emprunt russe avortait..." 47, Mercier doit se contenter d'un prêt à court terme de $\$ 4,000,000$ à un taux d'intérêt de $4 \%$. Ce sont le Crédit lyonnais et la Banque de Paris et des Pays-Bas qui lui accordent encore leur faveur et qui fixent la remise au 15 juillet $1893^{48}$. A cette date, le gouvernement est autorisé à rembourser les 40,000 obligations de 1891 par la création d'un nouvel emprunt à court terme, aux mêmes conditions qu'en 1891 et remboursable le 15 juillet 1895 . Le comte de Turenne, cette fois encore, essaie d'inciter le gouvernement français à refuser toute tentative de la part de Chapleau en le décrivant comme "un esprit sans consistance et d'un caractère mobile ${ }^{49 "}$. La transaction ne semble cependant avoir soulevé aucun problème en France ou à l'Assemblée législative du Québec.

En 1894, il est encore possible de recourir à la loi de 1890 permettant des emprunts successifs jusqu'à concurrence de $\$ 10,000,000$. En effet, la province a réalisé l'emprunt de 1891 qu'elle a renouvelé en 1893. Le trésorier Hall s'est procuré trois millions de dollars auprès de banques locales en mars 1894 et le premier ministre Taillon veut profiter de la somme qui reste à sa disposition. Son trésorier étant malade, Taillon administre le trésor par intérim et entame lui-même les négociations avec le Crédit lyonnais et la Banque de Paris et des Pays-Bas, dans le but de réaliser cette fois un emprunt à long terme. Ce dernier emprunt du Québec en France au cours du $19^{\circ}$ siècle, ou plutôt la transformation en un prêt à long terme de la transaction de 1893, provoque de fortes réactions à la législature québécoise et dans certains milieux financiers.

$46 \mathrm{CP}$, Angleterre 85, 274 et verso - 14 février 1891. Cité par M. Pierre Savard, "Les Canadiens français vus par les consuls de France à Québec et à Montréal de 1859 à 1900", RHAF, 21, no 2 (sept. 1967) : 227.

47 Robert Rumilly, Histoire de la province de Québec, VI: 229.

48 DSP, 1893, doc. no 53: 12.

49 AAE, Paris, $C C, 22$ mars 1893. Cité dans l'article de M. Pierre Savard, loc. cit., 227. 
C'est Edmond Barbeau, administrateur du Crédit foncier franco-canadien, filiale canadienne de la Banque de Paris et des Pays-Bas, qui est chargé d'aller négocier l'emprunt en France. Pendant ce temps, au Québec, les antagonismes locaux s'affrontent. En 1894, en effet, Londres et les banques canadiennes sont riches "en disponibilités" et c'est pour cette raison que la Banque de Montréal veut torpiller le projet. Le Pacifique Canadien et le trésorier de la province, Hall, serviront d'instruments à cette puissante institution. Le 19 juin 1894, le Pacifique Canadien offre de payer les $\$ 7,000,000$ qu'il doit au gouvernement pour l'achat du premier chemin de fer du Nord. Taillon fait cependant remarquer à son trésorier, qui appuie d'une part la thèse d'un emprunt à Londres ou au Canada et d'autre part la remise du Pacifique Canadien:
...qu'il n'est pas avantageux de réaliser la créance sur le Pacifique, puisque cette Compagnie verse ré- gulièrement, sur les 7 millions, un intérêt de 5 p. 100; que la loi passée lors de la cession du chemin de fer du Nord réserve les paiements du Pacifique à l'amortissement des emprunts de 1874 et 1876, con- tractés pour le chemin de fer. De sorte que les sept millions, ne pouvant servir ni aux dépenses cou- rantes, ni au remboursement de l'emprunt Mercier, ne résoudraient pas le problème ${ }^{50}$.

Le premier ministre continue donc les pourparlers avec Paris et conclut un emprunt à long terme de $\$ 4,000,000$, à $3 \%$ d'intérêt.

C'est à ce moment que l'opposition éclate. Le trésorier Hall démissionne en octobre 1894; les journaux anglais critiquent le premier ministre Taillon; la Banque de Montréal déclare qu'elle résistera par tous les moyens; le Pacifique Canadien retire son offre de remise et les émissaires de cette opposition inquiètent les banquiers français sur le sort de leur contrat devant la législature québécoise. Enfin, la question soulève les nationalismes. D'un côté la presse française appuie Taillon, de l'autre la presse anglaise favorise la Banque de Montréal, le Pacifique Canadien et Hall.

Lorsque la session provinciale ouvre en novembre 1894, on se demande si les 11 députés conservateurs canadiens-anglais appuieront leur parti ou la thèse de Hall. Ce n'est qu'après de longues discussions, publiques et secrètes, que le 6 décembre

50 Rumilly, op. cit., 188-189. 
suivant les ministériels reçoivent un vote majoritaire. Seulement six conservateurs canadiens-anglais votent du côté de l'opposition. La crise est donc conjurée et à la fin de décembre, l'accord sur l'emprunt devient final. Cet argent sera destiné exclusivement à la conversion et au remboursement des obligations de 1893 et devra être remis au plus tard le 20 janvier $1955^{51}$.

Ces emprunts du Québec en France resserrent, au cours de cette période, les liens entre les deux pays. Celui de 1881 revêt à nos yeux une importance particulière. Il peut en effet être considéré comme l'acte procréateur de l'initiative la plus intéressante dans l'histoire de ces investissements, comme la cause directe de la fondation du Crédit foncier franco-canadien au cours de cette même année. De plus, en 1882, on assiste à l'ouverture du commissariat général du Canada à Paris; Hector Fabre assume la tâche de commissaire et d'agent commercial et financier du Québec à Paris. En 1886, la Chambre de commerce française de Montréal est fondéc et participe à la création de la première liaison directe et régulière entre la France et le Canada, la Compagnie Bossière. A chacune des rencontres officielles qu'entraînent ces événements, nous remarquons que les orateurs, tant français que canadiens-français, considèrent l'année 1881, c'est-à-dire l'emprunt du Québec et la fondation du Crédit foncier franco-canadien, comme la poussée nécessaire qui a permis l'intensification des relations franco-québécoises au cours des années subséquentes.

\section{Le Crédit foncier franco-canadien}

$\mathrm{Au}$ milieu du $19^{\mathrm{e}}$ siècle, la France et l'Allemagne d'abord, la Suède, l'Autriche, l'Espagne, le Luxembourg et l'Egypte ensuite, produisent les premières sociétés de crédit foncier qui se développent sous la tutelle de chacun de ces gouvernements. En France, à partir du moment où en 1852 le gouvernement étend à tous les départements le privilège de la Banque foncière de Paris et lui donne le nom de Crédit foncier de France, l'histoire du crédit foncier français se confond avec celle de cette grande institution. D'autres pays cependant laissent à l'entreprise privée la charge de créer ces institutions qui rappelleront d'ailleurs de très près le type adopté en France. Le Crédit foncier francocanadien, fondé au Québec en 1880, sera d'ailleurs une création de l'entreprise privée.

51 DSP, 1894, 28, doc. no 14: 57-59. 
EMPRUNTS DE LA PROVINCE DE QUÉBEC EN FRANCE

\begin{tabular}{|c|c|c|c|c|c|c|c|c|c|}
\hline $\begin{array}{c}\text { Date } \\
\text { d'émission }\end{array}$ & Montant & Maturité & $\begin{array}{c}\% \\
\text { intérêt }\end{array}$ & $\begin{array}{c}\% \\
\text { prix de } \\
\text { vente } \\
\text { moins } \\
\text { intérêt } \\
\text { accumulé }\end{array}$ & $\begin{array}{l}\text { Escomptes } \\
\text { sur } \\
\text { obligations }\end{array}$ & $\begin{array}{c}\text { Intérêt } \\
\text { accumulé }\end{array}$ & $\begin{array}{c}\text { Escompte } \\
\text { au-dessous } \\
\text { du pair } \\
\text { sur change }\end{array}$ & Frais & $\begin{array}{c}\text { Somme } \\
\text { nette } \\
\text { réalisée }\end{array}$ \\
\hline$\underset{1880}{\text { 1er juillet }}$ & $\$ 4,275,853.34$ & $\underset{1919}{1^{\text {er juillet }}}$ & $41 / 2$ & $891 / 4$ & $\$ 460,386.67$ & & $\$ 42,749.44$ & $\$ 15,278.64$ & $\$ 3,757,438.59$ \\
\hline $15 \underset{1891}{\text { juillet }}$ & $\$ 3,860,000.00$ & 15 juillet & 4 & 96 & $\$ 152,470.00$ & $\$ 13,536.44$ & $\$ 14,115.19$ & $\$ 21,941.00$ & $\$ 3,657,937.37$ \\
\hline $15 \underset{1893}{\text { juillet }}$ & $\$ 4,106,461.00$ & 15 juillet & 4 & 94 & $\$ 246,387.66$ & & & $\$ 10,767.00$ & $\$ 3,849,306.27$ \\
\hline $\begin{array}{c}30 \text { décembre } \\
1894\end{array}$ & $\$ 5,332,976.00$ & $\begin{array}{c}20 \text { janvier } \\
1955 \text { ou } \\
\text { après le } \\
20 \text { janvier } \\
1905 \text { sous } 6 \\
\text { mois d'avis }\end{array}$ & 3 & 77 & $\$ 1,226,584.48$ & & & $\$ 1,587.53$ & $\$ 4,104,803.99$ \\
\hline
\end{tabular}


Depuis plusieurs années déjà, les agriculteurs du Québec s'orientent de plus en plus vers l'élevage et l'exploitation des produits laitiers. Depuis un bon nombre d'années aussi retentissent régulièrement à l'Assemblée législative des discours pathétiques tentant de convaincre le gouvernement de venir en aide à un secteur si essentiel à notre économie. En effet les agriculteurs attendent toujours les facilités de crédits nécessaires à la stimulation des nouvelles spécialisations.

En 1862, un consul français à Québec, Gauldrée Boileau, avait mentionné la nécessité de venir en aide aux régions rurales et attiré l'attention sur les rumeurs qu'une banque de crédit foncier serait fondée à St-Hyacinthe ${ }^{52}$. Il n'en fut rien. En 1880 cependant, lorsque J.-S.-C. Würtèle se rend à Paris afin de contracter un emprunt au nom de la province, il a aussi comme mission de poser les bases d'une institution de crédit foncier. Ce sont d'ailleurs les mêmes banquiers, ceux de la Banque de Paris et des Pays-Bas, qui négocient les deux affaires. Le Crédit foncier franco-canadien sera donc une succursale canadienne de cette grande institution. Les initiateurs au Québec sont le premier ministre Chapleau et le consul français Albert Lefaivre.

Le 24 juillet 1880, l'acte d'incorporation de la société est sanctionné par le lieutenant-gouverneur de la province aux noms du comte Raphaël-Maximilien Cahen d'Anvers, Edmond-Jean Joubert, Charles-Louis Sautter, tous trois de la Banque de Paris et des Pays-Bas, Joseph-Adolphe Chapleau, Etienne-Théodore Paquet, Jonathan-S.-C. Würtèle, Jean de Beaufort, Isidore Thibaudeau et de quelques autres. Selon cet acte, les objectifs de la compagnie sont

... de prêter sur hypothèque, aux propriétaires d'immeubles (...) de prêter sur la garantie de créances hypothécaires ou privilégiées (...) de prêter, avec ou sans hypothèque, aux corporations municipales et scolaires, aux fabriques et aux syndics pour la construction ou la réparation des églises (...) d'acquérir, par voie de subrogation ou de transport, des créances hypothécaires ou privilégiées sur des immeubles (...) de faire en un mot, toutes les opérations ayant pour but de développer les prêts sur les immeubles dans la province de Québec (...) de faire des prêts au gouvernement de la province... ${ }^{\text {s3 }}$

52 AAE, Paris, CC, tome 11, 22 décembre 1862, fol. 058.

53 DSP, 1880, attaché au premier rapport du Crédit foncier en 1881. 
Le fonds social de la société est fixé à $\$ 5,000,000$ divisés en 50,000 actions de $\$ 100$ chacune. En fait, la loi organique de juillet 1880 crée un bureau à Québec et l'autre à Montréal, mais cette dernière ville ne tarde pas à monopoliser le gros des transactions pour ainsi reléguer Québec au rang de simple succursale.

Les réactions, à la suite de cette fondation, semblent en général être favorables. Le consul Lefaivre, dans une lettre datée du 9 octobre 1880 aux autorités françaises, cite la plupart des journaux canadiens-français; ils sont tout à fait enthousiastes. Il se garde bien évidemment de tenir compte des journaux canadiens-anglais de Montréal, des milieux financiers qui affronteront cette concurrence, et plus tard de la réaction de certains membres de l'opposition gouvernementale. C'est d'ailleurs dans le but de renseigner le public sur le crédit foncier et de "dissiper les appréhensions des capitalistes habitués à prêter sur propriétés foncières, qui voient, dans la création du Crédit foncier franco-canadien, l'introduction d'un monopole injuste", que le gouvernement croit bon de publier sous forme de brochure une série d'articles favorables écrits par un certain L.-N. Carrier dans Le Canadien ${ }^{54}$. Enfin, dès l'ouverture de la session provinciale, le 28 avril 1881, le jour même où Chapleau commente la situation économique, l'arrivée des capitaux français et l'établissement du Crédit foncier franco-canadien, L'Electeur dénonce un scandale qui sera relevé par l'opposition au cours de plusieurs sessions subséquentes. Le journal déclare que Chapleau, Paquet et Würtèle auraient reçu chacun $\$ 14,000$ des banquiers français pour l'introduction du Crédit foncier au Québec. Par la suite, une enquête dévoile que seul Paquet aurait accepté l'argent offert.

Malgré l'existence de ces sentiments partagés sur l'introduction de cette société au Québec, les responsables de l'entreprise débordent d'enthousiasme et se félicitent des résultats obtenus avant même le début des opérations. Le consul français à Québec se charge d'annoncer la bonne nouvelle dans son pays :

Telles sont les espérances qu'inspire cette institution à l'agriculture que, dès à présent, c'est-à-dire avant l'ouverture des opérations, l'intérêt des prêts hypothécaires a baissé dans toute la province de 2 à $21 / 2$ p. 100 . La classe rurale éprouve un soulagement immense et c'est avec un véritable enthousiasme

54 Les institutions de crédit foncier. Quelques notes sur leur but, leur organisation et leur origine (Québec, Demers \& Frère, 1880), 10 et 28. 
qu'elle accueille l'annonce d'entreprises nouvelles, projetées par des industriels et capitalistes français ${ }^{55}$.

La direction du Crédit foncier franco-canadien est confiée, dès le départ, à Edmond-Julien Beaubien, administrateur de la Banque d'Epargne de la cité et du district de Montréal et receveur général du Canada dans cette même ville. Il est assisté de Martial Chevalier, fils de l'ancien consul général de France à Québec entre 1872 et 1875 . La compagnie devra d'ailleurs à ces deux hommes d'avoir surmonté les moments difficiles inhérents au lancement d'une telle entreprise.

En avril 1881, le Crédit foncier franco-canadien commence ses opérations. Dès le 31 décembre de la même année, le placement de la majeure partie du capital versé, soit de $6,000,000$ de francs, le quart des $25,000,000$ autorisés, est déjà effectué. Une transformation importante a cependant eu lieu au cours de ce premier terme. En effet, à peine constituée, la compagnie se voit menacée par la venue d'une rivale, la Société Kohn Reinach et Compagnie ou Société financière de Paris, concessionnaire de l'Intercolonial Pacific. Cette dernière obtient une charte fédérale qui annule l'exclusivité que le Crédit foncier franco-canadien avait obtenue par sa charte provinciale au Québec. Afin de lutter à armes égales avec leur concurrente, les administrateurs obtiennent donc eux aussi une charte fédérale et renoncent par le fait même au privilège que leur accordait le gouvernement du Québec en interdisant la venue d'autres compagnies à capitaux français sur le même territoire.

Les rapports annuels que la compagnie fournit à partir de 1882 décrivent donc la situation canadienne de l'entreprise. Toutefois, avant de présenter à l'aide de tableaux l'évolution du Crédit foncier franco-canadien, nous pensons qu'il serait judicieux d'apporter quelques considérations pouvant en faciliter l'interprétation. Il faut d'abord souligner qu'à partir de 1889 , à cause de la concurrence redoutable des compagnies d'assurances concernant les prêts sur hypothèque, l'activité de la société se porte principalement sur les prêts urbains, à intérêt simple, sans amortissement. Cela signifie que les prêts ruraux, à long terme, deviennent plus rares, que les régions rurales de Québec et de la Gaspésie se voient presque entièrement supplantées par les régions urbaines de Montréal et de Toronto.

55 AAE, Paris, CC, tome 9, 9 octobre 1880, fol. 81. 
Il nous faut aussi admettre que l'interprétation qu'on peut faire, les liens qu'on peut établir entre prêt urbain et court terme, entre prêt rural et long terme, doivent être sujets à caution. Au cours de la période $1881-1900$, ces relations semblent correspondre à une certaine réalité, c'est un fait, mais elles ne pourraient être applicables qu'à des régions comme celles de Québec, de la Gaspésie et des Maritimes en général. Il devient donc très difficile de préciser quelles sont les sommes qui ont été prêtées et consacrées au développement de l'agriculture québécoise, à la réorientation de cette agriculture et à certaines autres catégories d'individus ou de corps publics. Les tableaux qui suivent doivent donc être consultés en tenant compte de ces avertissements.

\section{ÉVOLUTION GÉNÉRALE}

\begin{tabular}{|c|c|c|c|c|c|c|}
\hline $\begin{array}{l}\text { Exer- } \\
\text { cices }\end{array}$ & Actif $(\$)$ & $\begin{array}{l}\text { Produits } \\
\text { bruts }(\$)\end{array}$ & $\begin{array}{l}\text { Bénéfices } \\
\text { nets }(\$)\end{array}$ & $\begin{array}{l}\text { Action } \\
\text { (Fr.) }\end{array}$ & $\begin{array}{l}\text { Part } \\
\text { (Fr.) }\end{array}$ & $\begin{array}{l}\text { Obligations en } \\
\text { circulation }(\$)\end{array}$ \\
\hline 1881 & $4,828,715.18$ & $53,442.00$ & $16,741.00$ & - & - & - \\
\hline 1882 & $877,022.52$ & & & 4.00 & & \\
\hline 1883 & & & & & & \\
\hline 1884 & & & & & & $1,076,500.01$ \\
\hline 1885 & & & & & & \\
\hline 1886 & & & & & & \\
\hline 1887 & & & & 6. & & \\
\hline 1888 & & & & & 1.278 & \\
\hline 1889 & 3.97 & 313 & & 7.50 & 2.13 & $4,329,884.76$ \\
\hline 1890 & 99.75 & 387, & & 8.00 & 2.98 & $7,735,604.53$ \\
\hline 1891 & 2.06 & 442, & & 8.00 & 2.98 & $7,145,417.94$ \\
\hline 1892 & $\overline{84.25}$ & 441 , & & 8.00 & 2.98 & $7,126,851.68$ \\
\hline 1893 & $12.288,780.46$ & & 80.00 & 8.00 & 2.98 & $7,065,653.00$ \\
\hline 1894 & $12,289,662.41$ & 3.00 & 59.00 & 8.00 & 2.98 & $7,021,017.21$ \\
\hline 1895 & 2.24 & & & 8.00 & 2.98 & \\
\hline 1896 & & & & 8.00 & 2.98 & \\
\hline $189^{\prime}$ & & & & & & \\
\hline & 12 & & & & & \\
\hline 1899 & $12,446,391.77$ & & & 8.00 & 2.98 & \\
\hline 1900 & $12,407,839.87$ & & & & 2.98 & \\
\hline 1901 & $12,398,457.25$ & $426,834.00$ & $92,267.00$ & 8.00 & 2.98 & $7,009,990.87$ \\
\hline
\end{tabular}

Les emprunts québécois en France et les opérations du Crédit foncier franco-canadien offrent aux investisseurs français, pendant ces vingt dernières années du $19^{\mathbf{e}}$ siècle, les deux seules occasions de réussite totale en territoire québécois. 
DISTRIBUTION DES PRETS

\begin{tabular}{|c|c|c|c|c|c|}
\hline \multirow[t]{2}{*}{ Exercice } & \multirow[t]{2}{*}{ Genre } & \multicolumn{2}{|c|}{ Canada } & \multirow[b]{2}{*}{$\underset{(\$)}{\text { Montréal }}$} & \multirow[b]{2}{*}{$\begin{array}{c}\text { Québec } \\
(\$)\end{array}$} \\
\hline & & $\begin{array}{l}\text { Prêts annuels moins } \\
\text { remboursements * } \\
(\$)\end{array}$ & $\begin{array}{c}\text { Prêts nouveaux } \\
\text { non cumulatifs } * *\end{array}$ & & \\
\hline \multirow[t]{2}{*}{1881} & L.T. & $674,716.33$ & \multirow[t]{2}{*}{$1,062,730.83$} & \multirow[t]{2}{*}{$514,741.99$} & \multirow[t]{2}{*}{$361,674.34$} \\
\hline & C.T. & $388,014.50$ & & & \\
\hline \multirow[t]{2}{*}{1882} & L.T. & $760,691.92$ & \multirow[t]{2}{*}{$140,933.67$} & $47,150.00$ & $29,800.00$ \\
\hline & C.T. & $410,252.71$ & & $1,800.00$ & - \\
\hline \multirow[t]{2}{*}{1883} & L.T. & $748,309.69$ & \multirow[t]{2}{*}{ - } & \multirow[t]{2}{*}{-} & \multirow[t]{2}{*}{ - } \\
\hline & C.T. & $405,729.90$ & & & \\
\hline \multirow[t]{2}{*}{1884} & L.T. & $1,011,187.97$ & \multirow[t]{2}{*}{$349,004.29$} & $203,300.00$ & $34,400.00$ \\
\hline & C.T. & $382,204.19$ & & $44,829.00$ & $8,900.00$ \\
\hline \multirow[t]{2}{*}{1885} & L.T. & $1,599,918.37$ & \multirow[t]{2}{*}{$753,268.00$} & $359,650.00$ & $123,250.00$ \\
\hline & C.T. & $434,118.82$ & & $30,800.00$ & $8,900.00$ \\
\hline \multirow[t]{2}{*}{1886} & L.T. & $1,959,210.88$ & \multirow[t]{2}{*}{$572,701.00$} & $239,751.00$ & $90,550.00$ \\
\hline & C.T. & $501,872.11$ & & $64,400.00$ & $3,400.00$ \\
\hline \multirow[t]{2}{*}{1887} & L.T. & $2,235,431.71$ & \multirow[t]{2}{*}{$813,994.70$} & $273,343.70$ & $61,350.00$ \\
\hline & C.T. & $811,680.37$ & & 209.976 .00 & $19,500,00$ \\
\hline \multirow[t]{2}{*}{1888} & L.T. & $2,307,451.96$ & $1,437,299.33$ & - & - \\
\hline & C.T. & $1,755,623.70$ & & & \\
\hline 1889 & L.T. & $2,473,768.84$ & $1,497,909.31$ & - & - \\
\hline & C.T. & $2,604,715.09$ & & & \\
\hline 1890 & L.T. & $2,507,204.59$ & $1,649,220.41$ & $232,447.00$ & $27,050.00$ \\
\hline & C.T. & $3,657,090.84$ & & $912,875.00$ & $6,214.51$ \\
\hline 1891 & L.T. & $2,413,398.46$ & $922,469.03$ & $152,910.00$ & $12,600.00$ \\
\hline & C.T. & $4,045,563.45$ & & $544,450.00$ & $13,000.00$ \\
\hline 1892 & L.T. & $2,291,226.91$ & $937,625.41$ & $127,160.50$ & $21,500.00$ \\
\hline & C.T. & $4,212,191.69$ & & $609,950.00$ & - \\
\hline 1893 & L.T. & $2,133,979.55$ & $1,273,977.50$ & - & - \\
\hline & C.T. & $4,780,854.90$ & & $* * *$ & $* * *$ \\
\hline 1894 & L.T. & $1,955,736.13$ & $932,875.69$ & - & - \\
\hline & C.T. & $4,999,271.22$ & & & \\
\hline 1895 & L.T. & $1,906,548.70$ & $716,005.88$ & - & - \\
\hline & C.T. & $5,121,300.95$ & & & \\
\hline 1896 & L.T. & $1,732,319.77$ & $860,656.56$ & - & - \\
\hline & C.T. & $5,214,953.16$ & & - & \\
\hline 1897 & L.T. & $1,736,969.97$ & $1,393,827.37$ & - & - \\
\hline & C.T. & $5,214,952.16$ & & & \\
\hline 1898 & L.T. & $1,583,486.04$ & $934,093.56$ & - & - \\
\hline & C.T. & $5,328,391.92$ & & - & \\
\hline 1899 & L.T. & $1,420,964.62$ & $1,510,095.56$ & - & - \\
\hline & C.T. & $5,651,556.63$ & & & \\
\hline 1900 & L.T. & $1,207,537.50$ & $654,974.96$ & - & - \\
\hline & C.T. & $5,712,913.74$ & & & \\
\hline 1901 & L.T. & $1,072,423.99$ & $842,183.82$ & - & - \\
\hline & C.T. & $5,547,743.11$ & & & \\
\hline
\end{tabular}

* Chiffres cumulatifs.

* Long et court termes.

*** A partir de 1893, on ne distingue plus entre Québec et Montréal. 
Les emprunts d'abord tirent le gouvernement de notre province de situations embarrassantes. Ils lui permettent même, dans la dernière décennie du siècle, d'afficher une certaine indépendance vis-à-vis des milieux financiers canadiens-anglais de Montréal, Toronto, et aussi vis-à-vis du marché londonien.

Le Crédit foncier, pour sa part, prête environ $\$ 20,000,000$ aux contribuables canadiens. Les données que nous possédons ne nous permettent pas d'établir la part exacte qu'il faut accorder au Québec dans cet ensemble canadien. Elles ne nous permettent pas, non plus, ce qui est encore plus déplorable, de fournir des approximations sur les prêts consentis respectivement aux cultivateurs, aux corporations municipales et scolaires et même au gouvernement de notre province.

Les chiffres que nous possédons, concernant les prêts accordés par le Crédit foncier aux Québécois, nous offrent la possibilité pourtant de formuler une hypothèse. Au cours des neuf années que nous pouvons considérer grâce à ces chiffres, la compagnie brasse près de $75 \%$ de ses affaires canadiennes au Québec, soit un montant de $\$ 5,392,623.04$ sur un total de $\$ 7,201,947.34$. Et même si cette proportion subit peut-être une légère baisse à la fin du siècle à cause de l'importance accordée au développement de l'Ouest et à la création de succursales dans ces régions, le Québec, de 1881 à 1901, demeure quand même le champ le plus propice aux opérations de la compagnie, nous ne pouvons en douter.

\section{4-LE SUCRE DE BETTERAVE LES PHOSPHATES}

Au cours de cette période où le Québec consacre une grande partie de ses efforts à la réorientation de son économie, la fabrication du sucre de betterave et l'exploitation des mines de phosphate attirent, pendant quelques années, l'attention des autorités provinciales. Certains entrepreneurs français, profitant de leur expérience dans ces domaines, veulent jouer un rôle d'initiateurs et espèrent ainsi se tailler une place avantageuse dans ces secteurs de notre économie.

\section{Le sucre de betterave}

Même avant la deuxième moitié du $19^{e}$ siècle, certaines autorités gouvernementales avaient pensé à la possibilité d'implanter au Canada des usines de transformation pour la betterave à sucre. "Elle était toutefois restée, pendant près de 50 ans, à 
l'état de problème ${ }^{56}$." Lorsque Edouard Barnard, au service du gouvernement fédéral, retourne en Europe en $1872^{57}$, il doit cependant se renseigner auprès des experts sur tous les détails concernant ce domaine. Et dans le rapport qu'il envoie quelques semaines plus tard, avertissant d'abord que la saison de 1872 est trop avancée pour envoyer des spécialistes français dans l'Ouest du Canada, il conseille au gouvernement de faire des essais dans différentes parties du pays afin de s'assurer de la richesse en saccharine de ce produit. Il exprime enfin l'opinion que le climat d'hiver des provinces de Québec et du NouveauBrunswick serait très favorable à cette culture ${ }^{58}$.

Entre 1872 et 1880 , le gouvernement du Québec incite les conférenciers agricoles à populariser cette idée dans les assemblées de cultivateurs, et offre des primes d'encouragement de $\$ 25,000$ en 1874 , de $\$ 70,000$ en 1875 , à la première fabrique de sucre de betterave qui s'installera dans la province.

Il encourage, surveille les expériences de culture et distribue aux volontaires un Traité populaire sur la culture de la betterave et la fabrication du sucre en Canada, écrit par Octave Cuisset de Québec en $1876^{59}$. Enfin, en 1877, Edouard Barnard peut fournir au gouvernement un rapport favorable sur la teneur en saccharine de ce produit québécois.

En 1879, la nouvelle politique tarifaire inaugurée par Ottawa affecte directement le volume et la qualité des importations de sucre au Canada et en même temps l'avenir des sucreries canadiennes et québécoises. Cette loi vise à hausser les tarifs sur le sucre raffiné, à encourager l'importation de sucres bruts et la création de raffineries sur notre territoire. Le tableau qui suit nous en montre les effets immédiats :

Si nous établissons que tous les sucres au-dessus de la catégorie 14 ont déjà subi un certain raffinage, nous constatons que nos industries ont 94,133,208 livres de produits à transformer en

56 Edouard Barnard, L'agriculture au point de vue de l'émigration et l'immigration (Montréal, Presse à vapeur de La Minerve, 1872), $8 \mathrm{p}$.

57 Pour plus de détails, voir l'ouvrage de Marc-André Perron, Un éducateur agricole. Edouard-A. Barnard (1835-1898). Essai historique sur l'agriculture, 1760-1900. Thèse de DES (Université Laval, déc. 1955), XXI$355 \mathrm{p}$.

58 Documents de la Session [fédérale] (DSF), 1892, 25 (5), doc. no 7c: 24-25. C'est M. Saunders, directeur des fermes expérimentales "de la puissance" qui cite ce rapport de M. Barnard.

${ }^{59}$ Ce fait est signalé dans le DSF, 1876, vol. 10, doc. no 3, app. no 2 : 53. Nous n'avons pu cependant trouver cette brochure. 
IMPORTATIONS CANADIENNES DE SUCRES

\begin{tabular}{l|c|c|c|c}
\hline & \multicolumn{2}{|c|}{$1878-1879$} & \multicolumn{2}{c}{$1879-1880$} \\
\cline { 2 - 5 } Catégories & (lbs) Quantité & $\%$ & (lbs) Quantité & $\%$ \\
\hline $\begin{array}{l}\text { Mélado devant } \\
\text { être raffiné }\end{array}$ & $1,628,439$ & - & $7,039,332$ & - \\
\hline Moins de 9 & $1,884,818$ & 1.68 & $30,690,625$ & 28.95 \\
\hline 9 à 14 & $19,390,746$ & 17.12 & $56,403,251$ & 53.22 \\
\hline Plus de 14 & $91,828,152$ & 81.20 & $18,885,150$ & 17.83 \\
\hline
\end{tabular}

1879-80 comparativement à 22,904,003 pour l'année précédente. Cela signifie que nos importations de sucres bruts provenant des Antilles anglaises et des îles espagnoles prennent rapidement le pas sur les sucres raffinés venant des Etats-Unis et de l'Angleterre. A Montréal et à Halifax, à cause de cette nouvelle situation, les usines de transformation augmentent leur production, la concurrence devient plus franche sur notre territoire et quelques industriels pensent même que l'utilisation de notre propre matière première, la betterave à sucre, pourrait entraîner une réussite certaine.

\section{L'Union sucrière du Canada}

En 1880, pour encourager les initiatives privées, le gouvernement canadien exempte de taxation, pendant les cinq premières années de leur existence, toutes les compagnies de sucre de betterave qui s'installeront au Canada. Au cours de la même année, des entrepreneurs français décident de profiter de cette conjoncture favorable et négocient avec les autorités fédérales la charte de l'Union sucrière du Canada. Il s'agit d'une société dont les $9 / 10$ du capital $(\$ 2,000,000)$ seront souscrites en France et le reste au Canada.

Les responsables montrent beaucoup d'enthousiasme au départ. Ils ont en effet l'intention d'installer des fabriques et des raffineries à Longue-Pointe, à Charlesbourg, à Berthier et à Varennes, comté de Verchères. En fait, le troisième de ces projets seulement fera l'objet d'une tentative. La compagnie signe quelques contrats avec des cultivateurs aux environs de Berthier et s'assure ainsi la culture de 1,636 arpents de betteraves. Au premier juillet 1881, les actionnaires français et canadiens versent respectivement $\$ 140,000$ et $\$ 60,000$. Au cours des mois 
de juillet, août et septembre, la machinerie importée de France au coût de $\$ 85,000$ est installée et la fabrique, à cette date, peut traiter environ 200 tonnes de betteraves par 24 heures.

Cependant, dès la première saison, l'expérience s'avère être un échec. La compagnie connaît des difficultés financières, les actionnaires ne veulent plus souscrire et les intérêts passent en d'autres mains à la fin de 1882. Plusieurs causes expliquent cette faillite: le manque d'entente administrative; l'insuffisance de capital; l'ensemencement trop tardif et la perte de plusieurs tonnes de betteraves qui gèlent ou pourrissent avant d'être utilisées. Elles n'expliquent toutefois que l'échec particulier de cette entreprise. Dans une brochure qu'il publie en $1886^{60}$, E. Agostini nous fournit une explication beaucoup plus profonde du peu de rentabilité et de la faillite de plusieurs expériences semblables au Québec. Il insiste surtout sur le manque de préparation du cultivateur, sur son manque d'intérêt et de méthode. Il aurait fallu, pour réussir dans ce domaine, s'attaquer à la culture même, labourer profondément, mettre des engrais en quantité et faire le sarclage avec soin, autant de détails très importants pour qui veut s'adonner à l'agriculture intensive. En un mot, il aurait fallu éduquer l'agriculteur québécois. En 1894, commentant les mêmes expériences faites au Québec quelques années plus tôt, la Chambre de commerce française de Montréal tiendra les mêmes propos ${ }^{61}$.

L'expérience de Berthier tentée par l'Union sucrière du Canada n'est pas le seul exemple de la faillite de capitaux étrangers investis dans la culture et le traitement de la betterave à sucre au Québec. Elle est cependant la seule compagnie à capitaux français qui ait fait une telle tentative. Au cours de cette période d'ailleurs, le sucre de betterave doit faire face à une concurrence très forte sur le marché international. Le sucre de canne lui cause des torts considérables et décourage la venue de nouveaux investissements dans ce domaine. Enfin, une cause bien particulière au Québec explique la diminution rapide de l'importance qu'on semblait attribuer à cette industrie au cours des années 1880 . On avait déjà découvert à ce moment-là les aptitudes du Québec pour l'industrie laitière; la réorientation de notre économie était déjà bien amorcée. Il est tout à fait normal, à cause de cette situation, qu'on ait attaché moins d'importance à d'autres expériences dont la réussite semblait douteuse.

${ }^{60}$ La France et le Canada. Agriculture, industrie, commerce. Rapport au Syndicat maritime et fluvial de France (Paris, 1886), 34.

61 Bulletin de la CCFM (décembre 1894). 


\section{Les phosphates}

Les vastes couches de phosphate de chaux dont on a constaté l'existence dans les provinces de Québec et d'Ontario, sont d'une importance très grande pour l'agriculture du Canada; et l'on peut en dire autant du fait que la pyrite de fer se trouve à proximité de ces dépôts, vu que cette circonstance donne les moyens de manufacturer le superphosphate à bas prix et de produire ainsi aisément un engrais prêt pour le sol ${ }^{62}$.

Nous avons emprunté ces remarques à J. H. Hope, ministre de l'agriculture au gouvernement fédéral. Il explique par la suite qu'au Canada, particulièrement au Québec et en Ontario, il n'est plus temps de partir à la conquête de terres nouvelles, mais d'utiliser les engrais et d'éviter l'épuisement de notre sol. Cette présence de couches de phosphate offre donc une occasion unique et bon marché de commencer le processus d'exploitation.

Il existe déjà en 1879 au Canada quelques compagnies anglaises, canadiennes et allemandes qui exploitent les phosphates et les exportent aux Etats-Unis et en Europe. Il semble cependant que la découverte de couches de phosphate dont nous parle M. Hope, au nord de l'Outaouais, entre la rivière Lièvre et Gatineau, soulève l'enthousiasme de quelques autorités fédérales et provinciales. Et au Québec en particulier, au moment où revient à la surface le projet d'une ligne transocéanique directe entre la France et le Québec, au moment où on cherche un fret lourd, constant et certain pour alimenter cette ligne, l'événement arrive à point.

Le Québec exporte déjà depuis quelques années une certaine quantité de moutons vivants en France. Dans une lettre qu'il adresse au premier ministre Chapleau, Foursin Escande, qui surveille l'exportation de ce bétail et accompagne un chargement de 500 moutons en 1879 , souligne quelques points intéressants. Il montre d'abord comment ces animaux, dans les conditions de l'époque, doivent faire le voyage vers Liverpool, y subir une quarantaine, être transportés de Liverpool à Weymouth par chemin de fer pour ensuite traverser la Manche vers Cherbourg. Il note qu'il faudrait éviter ces transbordements qui font mourir les moutons ou les font parvenir sur le marché de Paris en très mauvaise condition physique. Il faudrait donc penser à une

62 DSF, 1879, 12 (7), doc. no 9: XII. 
exportation directe entre Québec et Cherbourg, et profiter de la découverte de phosphates pour fournir à la nouvelle ligne transocéanique son fret le plus important.

Foursin Escande, une fois à Paris, se met en relation avec Charles de Molon, spécialiste en matière d'engrais et de science agricole et attaché au ministère du commerce et de l'agriculture du gouvernement français. Ce dernier se montre intéressé et pose comme condition que le phosphate soit exporté à l'état brut, du Québec, et traité en France, alléguant que les produits nécessaires à la transformation ne se trouvent pas en territoire québécois. En avril 1880, le gouvernement du Québec acquiesce,

...considérant les avantages importants que la pro-
vince peut retirer de l'exportation régulière du bétail
vivant et des produits agricoles sur les marchés de
France, et dans le but de faciliter la création d'une
ligne de steamers entre le Canada et la France, consi-
dérant que le commerce sera en général avantagé,
considérant la subvention que le gouvernement pro-
vincial peut compter du gouvernement fédéral pour
une telle ligne....3

Que se passe-t-il exactement par la suite? Charles de Molon essaie de régler la question de la subvention annuelle de $\$ 10,000$ que le gouvernement fédéral offre pour la création de la ligne transocéanique. Il est d'ailleurs aidé par le premier ministre du Québec et $M$. Lefaivre, consul général de France à Québec. Les conditions sont posées et les intéressés semblent d'accord. En mars 1881 cependant, lorsque le Québec vend les terrains riches en phosphate à M. de Molon, ce dernier ne remplit pas les conditions de l'enchère. Quelques mois plus tard, le commissaire des terres de la couronne, E. J. Flynn, annule la transaction et l'acheteur perd les $\$ 3,000$ déposés au cours de l'encan. Comment expliquer ce manque d'intérêt soudain de la part de ce dernier? C'est que les financiers français, avec lesquels Charles de Molon faisait affaire, semblent attacher beaucoup plus d'importance à la création de la ligne directe transocéanique entre la France et le Canada qu'à l'exploitation des phosphates au Québec. La lutte s'engage donc entre différents concurrents auprès du gouvernement fédéral au sujet des subventions promises; $M$. de Molon perd la confiance de ses appuis financiers et laisse tomber son projet.

${ }^{63}$ DSP, 1882, 15 (2), doc. no 28. 


\section{La Société française des phosphates du Canada}

Selon Frédéric Gerbié ${ }^{64}$, une autre tentative aurait été faite par des entrepreneurs français dans ce domaine. Ils auraient en effet acheté en 1882 environ 6,000 acres de terres à phosphate, et commencé l'exploitation. Obalski, ingénieur minier au service du gouvernement québécois, confirme ce fait: "Cette compagnie, ayant ses centres principaux dans les environs du lac Tamo, a cessé ses travaux depuis deux ans, après avoir extrait près de 2,000 tonnes...65" M. Robert Rumilly, pour sa part, dans son troisième tome sur l'histoire de la province de Québec, nous éclaire sur les circonstances de la formation. Il affirme qu'à la suite du premier emprunt du Québec en France et de la création du Crédit foncier franco-canadien en 1880, on avait pensé compléter ces transactions par l'établissement d'une ligne de navigation directe et régulière avec la France. Un nommé Lalonde, bordelais entreprenant, faisant partie d'une mission française au Canada, aurait provoqué, à son retour en France, "la formation d'une seconde compagnie pour l'exploitation des phosphates canadiens...6"'. Un manque d'unité administrative, une insuffisance de capital, la perte d'une partie de l'argent investi et le choix d'un terrain de mauvaise qualité auraient forcé la Société française des phosphates du Canada à cesser ses opérations. Le manque de documents nous empêche d'aller plus avant au sujet de cette tentative.

En fait, l'exploitation des phosphates canadiens, au cours de cette période, ne constitue pas une expérience très heureuse pour le pays entier. De 1878 à 1885, la production canadienne passe de 3,000 à 25,000 tonnes et se maintient à ce niveau jusqu'au début des années 1890. La presque totalité de notre production est exportée en Angleterre. Mais en 1890-91, des gisements très importants de ce produit sont découverts en Floride. La concurrence américaine rend la continuation de la production canadienne d'abord presque impossible, et nulle ensuite à partir de 1893.

La concurrence de la Floride, dans le domaine des phosphates, nous offre un exemple frappant de l'interaction régionale à l'intérieur d'une économie qui devient continentale. Dans un tel contexte, la spécialisation d'une région économique du Canada doit tenir compte, non seulement des autres parties de ce pays, mais aussi de la concurrence qu'elle devra rencontrer sur tout le continent américain.

${ }^{64}$ Le Canada et l'émigration française, 368.

65 DSP, 1886, 19 (1), doc. no 4, app. 40.

66 Histoire de la province de Québec, III: 27-28. 


\section{CONCLUSION}

Il est tout à fait normal, après cette étude, de vouloir évaluer globalement la somme des capitaux français investis au Québec entre 1850 et 1900 . Il est possible, même si cette méthode risque de choquer les esprits avides d'exactitude, de parvenir à certains résultats par approximations successives. Nous avons formulé une hypothèse concernant le total des prêts consentis par le Crédit foncier franco-canadien au Québec, entre 1881 et $1900^{67}$. Elle nous fournit le montant le plus élevé de cette addition, soit une somme d'environ $\$ 15,000,000$. Ce chiffre pourrait comprendre les prêts hypothécaires à court et à long terme, les créances hypothécaires, les capitaux nécessaires à la rémunération du personnel et à la location des locaux de Montréal et de Québec. Les deux prêts à long terme accordés au gouvernement de la province de Québec en 1880 et 1894 par la Banque de Paris et des Pays-Bas, les frères Cahen d'Anvers et le Crédit lyonnais, nous permettent ensuite d'ajouter près de $\$ 8,000,000$ à ce tableau. Enfiñ, il n'est que raisonnable de placer comme dernier terme de cette addition quelques centaines de milliers de dollars pour rendre justice aux responsables des expériences plus ou moins heureuses de Berthier, Metgermette, lac Mégantic et de l'Outaouais. Cela signifie que les capitalistes français ont investi au Québec environ $\$ 23,500,000$ au cours de la deuxième moitié du $19^{\mathbf{e}}$ siècle.

Mais ce chiffre n'a de valeur significative, en autant qu'on puisse l'associer au financement de réalisations économiques de première importance pour cette même période, dans la province de Québec. Considérons chacune des expériences décrites dans l'ordre où nous les avons traitées. Celles de Metgermette et de la région du lac Mégantic se rattachent à deux domaines de caractère quasi sacré: la conquête de terres neuves et l'exploitation forestière. Nous ne prétendons pas attribuer à la première de ces expériences un impact considérable sur l'économie québécoise. Elle a toutefois contribué à la création d'un centre d'intérêt et au défrichement de terres qui ont servi à l'établissement d'un groupe de Canadiens français, à partir de 1884. La création de la Compagnie de colonisation et de crédit des Cantons de l'Est a entraîné la réalisation de travaux dont les conséquences nous semblent plus palpables, même encore au $20^{\circ}$ siècle. Il faut en effet reconnaître à cette entreprise le mérite de la création du village de Channay pour l'exploitation forestière et le déve-

${ }^{67}$ Hypothèse selon laquelle le Crédit foncier consent $75 \%$ de ses prêts dans la province de Québec, entre 1881 et 1900 . 
loppement de l'agriculture. Elle fut aussi à l'origine de l'organisation et l'exploitation du Moulin Nantais et de la venue d'un nombre important de travailleurs et de colons sur les bords de la rivière de l'Arnold et du lac Mégantic. Il faut aussi reconnaître que, même après la liquidation de 1890 , les bases solides posées grâce à l'initiative française ne se sont pas écroulées. Elles sont passées en d'autres mains et ont continué à être un facteur économique essentiel au développement de cette région.

Il est relativement plus facile de mesurer ou plutôt d'imaginer les effets d'une injection de près de $\$ 8,000,000$ dans le trésor d'une province qui a besoin de parachever son réseau ferroviaire, ou de réorienter différents secteurs de son économie. Les prêts français de 1880 et 1894 accordés à la province de Québec ont pu jouer un tel rôle.

Le premier de ces emprunts, celui de 1880 , a provoqué la création du Crédit foncier franco-canadien. Il était d'ailleurs tout à fait logique que la province de Québec, pour prouver sa reconnaissance, permette aux banquiers français d'ouvrir une succursale à l'intérieur de nos frontières. Le consul Lefaivre brosse-t-il un tableau de la réalité en 1880, lorsqu'il affirme que l'emprunt français et la fondation du Crédit foncier ont relancé notre économie et permis aux cultivateurs de revivre? En fait, il décrit la reprise économique des années 1880-1882 et attribue aux capitaux français une responsabilité beaucoup trop exclusive de la chose. Il est en tout cas certain que le Crédit foncier, entre 1881 et 1900 , a mis une quinzaine de millions de dollars à la disposition des Québécois. Il est aussi agréable de penser qu'au moment où la classe rurale oriente ses efforts vers la production et l'exploitation des produits laitiers, qu'au moment où elle a besoin d'investir dans ce domaine, cette institution française ait pu lui accorder, à un taux raisonnable, une partie, quelle que soit son importance, des crédits nécessaires à toute transformation.

Les tentatives françaises dans l'exploitation des phosphates et de la betterave sucrière ont été beaucoup moins fructueuses. Les capitaux français ont cependant fait figure de pionniers dans ces domaines au Québec. Ils ont ainsi poussé d'autres intérêts étrangers à y investir certaines sommes et à tenter des expériences enrichissantes pour les cultivateurs et les agronomes de nos régions.

Ce mouvement des capitaux français vers le Québec s'est effectué à l'intérieur d'une période à la fois précise et très restreinte, qui se situe au tout début des années 1880 . Pourquoi? La reprise économique tant attendue depuis la crise internatio- 
nale bancaire de 1873 coïncide avec le début de cette décennie. $\mathrm{Au}$ cours de ces mêmes années, en France, des institutions financières de première importance se livrent une lutte acharnée. Elle provoquera d'ailleurs la faillite de l'Union générale, en février 1882, et entraînera une débâcle financière retentissante. Cette lutte, qui se déroule surtout à partir de 1880, fait baisser les taux d'intérêt en France et provoque une fuite de capitaux vers l'étranger. Le Québec, qui connaît une courte période de prospérité à ce même moment, en attire une mince part. Nous pensons devoir accorder une certaine importance à ces facteurs.

Nous devons à Albert Lefaivre, consul général de France à Québec, de 1875 à 1881, d'avoir permis aux deux régions de profiter des conjonctures économiques favorables. En 1876, sous son influence, un publiciste français, M. de Molinari, visite plusieurs provinces du Canada. Au retour, ce dernier travaille, auprès des financiers français, à présenter le Québec comme un champ profitable à leurs activités. M. Lefaivre prépare les esprits des deüx côtés de l'océan et avertit le gouvernement et les financiers français du moment propice aux investissements. En novembre 1880, au cours d'un banquet donné à Montréal en l'honneur de délégués français, tous vantent justement ses mérites. Joseph Taché le présente comme l'homme "intelligent et sympathique initiateur de presque toutes les entreprises qui vont s'implanter au milieu de nous ${ }^{68}$ ".

Les remarques d'Armand Yon, même si elles ne s'appliquent pas strictement à l'économique, méritent quelque considération. Pour lui, la période 1830-1880 en est une de sentimentalité dans les relations culturelles franco-québécoises; période au cours de laquelle on invoque surtout la fidélité des Canadiens français aux traditions de la mère patrie. "C'est seulement vers 1880 (...) qu'on verra s'instaurer une période nouvelle, où le peuple canadien sera passé au crible d'une critique fréquente, souvent injuste, parfois salutaire ${ }^{69 . " ~ N o u s ~ p o u r r i o n s ~ p e u t-e ̂ t r e ~ e ́ t a b l i r ~ u n e ~ c e r-~}$ taine relation entre les descriptions trop idylliques des années antérieures à 1880 et l'hésitation des capitalistes français, ces hommes qui ont besoin de miser sur la réalité, à risquer un tant soit peu sur notre territoire.

${ }^{68}$ La France et le Canada français. Discours prononcés au banquet le 18 nov. 1880 par les citoyens de Montréal à MM. Thors, de Molinari et de Lalonde, délégués français (Montréal, Ateliers de La Minerve, 1880), VII. 69 Armand Yon, "Les Canadiens français jugés par les Français de France 1830-1939", RHAF, 18, no 3 (décembre 1964): 342. 